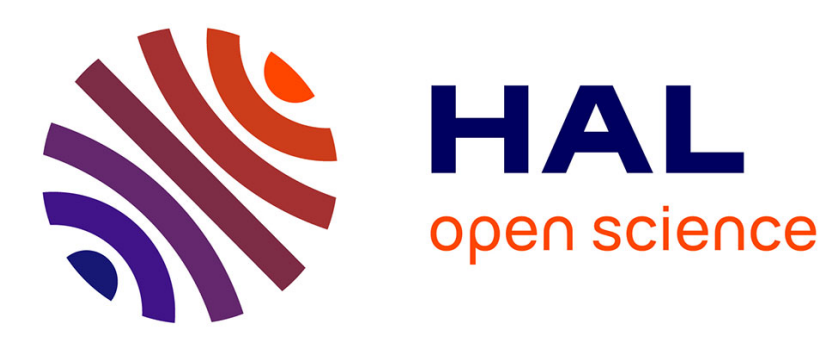

\title{
Multiscalar DIC Analyses of Granular String Under Stretch Reveal Non-standard Deformation Mechanisms
}

Nima Nejadsadeghi, Michele de Angelo, Anil Misra, François Hild

\section{To cite this version:}

Nima Nejadsadeghi, Michele de Angelo, Anil Misra, François Hild. Multiscalar DIC Analyses of Granular String Under Stretch Reveal Non-standard Deformation Mechanisms. International Journal of Solids and Structures, 2022, 239-240, pp.111402. 10.1016/j.ijsolstr.2021.111402 . hal-03482296

\section{HAL Id: hal-03482296 https://hal.science/hal-03482296}

Submitted on 15 Dec 2021

HAL is a multi-disciplinary open access archive for the deposit and dissemination of scientific research documents, whether they are published or not. The documents may come from teaching and research institutions in France or abroad, or from public or private research centers.
L'archive ouverte pluridisciplinaire HAL, est destinée au dépôt et à la diffusion de documents scientifiques de niveau recherche, publiés ou non, émanant des établissements d'enseignement et de recherche français ou étrangers, des laboratoires publics ou privés. 
1 Highlights

2 Multiscalar DIC Analyses of Granular String Under Stretch Reveal Non-standard Deformation Mechanisms

4 Nima Nejadsadeghi,Michele De Angelo,Anil Misra,François Hild

6

7

8

- DIC analyses with a suite of multiscalar kinematic assumptions are applied to measure displacement fields in a grain-string under extension

- Analyses reveal non-standard deformation mechanisms that underlie the emergent macroscale response

- Multiscale DIC analyses can elucidate otherwise latent mechanisms that indicate the need for refined kinematic descriptions 


\title{
Multiscalar DIC Analyses of Granular String Under Stretch Reveal Non-standard Deformation Mechanisms
}

\author{
Nima Nejadsadeghi ${ }^{a}$, Michele De Angelo ${ }^{b, c}$, Anil Misra ${ }^{a, b}$ and François Hild ${ }^{d, *}$ \\ ${ }^{a}$ Mechanical Engineering Department, University of Kansas, 1530 W 15th Street, Learned Hall, Lawrence, KS \\ 66047-7609, USA \\ ${ }^{b}$ Civil, Environmental and Architectural Engineering Department, University of Kansas, 1530 W 15th Street, \\ Learned Hall, Lawrence, KS 66047-7609, USA \\ ${ }^{c}$ Dipartimento di Ingegneria Civile, Edile-Architettura e Ambientale, Università degli Studi dell'Aquila, Via Giovanni \\ Gronchi 18 - Zona industriale di Pile, 67100 L'Aquila, Italy \\ ${ }^{d}$ Université Paris-Saclay, ENS Paris-Saclay, CNRS, LMT - Laboratoire de Mécanique et Technologie, 4 avenue des \\ sciences, 91190 Gif-sur-Yvette, France
}

\section{ARTICLE INFO}

Keywords:

Chirality

Digital Image correlation (DIC)

Granular material

Microstructured solid

Metamaterial

\section{A B S T R A C T}

Mechanical tests designed to measure classical behavior do not provide information underlying the macroscale response. Multiscalar digital image correlation (DIC) can be employed in these experiments to expose the micromechanisms that lead to the emergent macroscale behavior. A multiscalar DIC methodology is applied to study the extensional behavior of a particular granular string with chiral properties. DIC was performed at macroscale in which the underlying microstructure is overlooked and the construct is treated as a continuum bar. Further, the underlying microstructure was exploited in a microscale DIC analysis to expose the rich deformation regimes. In addition, mesoscale analysis was carried out to extract the rigid body motions that characterize a granular material system. These analyses were performed for two types of surface patterns and measurement uncertainties were quantified. The microscale results show that the grain string can be treated as a granular material composed of a set of nearly rigid grains that store elastic energy through intergranular mechanisms. Further, the results reveal non-standard deformation mechanisms that underlie the chiral granular string subjected to extension. These mechanisms include grain rotations that are coherent as opposed to gear-like grain rotation. More notably, grain transverse displacements are observed that are controlled by the behavior of the grain interconnections.

\footnotetext{
*Corresponding author.

francois.hild@ens-paris-saclay.fr (F. Hild); francois.hild@ens-paris-saclay.fr (F. Hild) ORCID(s): 0000-0002-2890-6483 (N. Nejadsadeghi); 0000-0002-4216-7525 (M. De Angelo);
} 


\section{Introduction}

Emergent response at the macroscale not only depends upon the microstructure, but also the micromechanics of interactions between micro-units. In many materials, these micromechanisms are latent and are not easily discoverable. To illustrate this phenomena, a granular microstructure was designed. This structure consists of a string of connected grains, whose connections are endowed with a particular behavior that couples tangential and normal grain-pair responses (Giorgio et al., 2020; Misra et al., 2020), thereby resulting in a chiral behavior that is not invariant to coordinate inversion. In other words, the mirror image of the geometry cannot be mapped onto itself by mere translations and rotations. The dynamics of chiral lattices has extensively been studied in the literature due to their peculiar phononic properties. In contradistinction to the chirality observed in wave transmission, as in optics, static deformation of solids may also exhibit a chiral behavior. A number of works have described chiral behaviors of lattice-like constructs by direct simulation of the microstructures using classical continuum theories (Alderson et al., 2010; Dirrenberger et al., 2011). On the other hand, experimental results have suggested the use of generalized continuum theories to correctly predict chirality. This observation has led several researchers to describe the complex deformation of chiral materials adopting generalized continuum theories (e.g., see (Spadoni et al., 2009; Liu et al., 2011, 2012; Chen et al., 2014; Frenzel et al., 2017; Duan et al., 2018; Poncelet et al., 2018; Reasa and Lakes, 2019; Wu et al., 2019; Biswas et al., 2020; Chen et al., 2020) to name a few).

In this work, the studied granular string design was inspired by micromorphic models of granular materials based upon granular micromechanics (Nejadsadeghi and Misra, 2020). Metamaterials, whose behavior is expected to be tailored to achieve some desired function, can benefit from rational design approach based upon predictive theories (dell'Isola et al., 2020). In this regard, the recent design and fabrication of pantographic metamaterials based upon higher gradient theories, and chiral granular materials are exemplar of such rational design (dell'Isola et al., 2019; Misra et al., 2020). These recent works have shown that theoretical methods using approaches such as 
Multiscale DIC can be implemented to reveal micromechanisms that may not be clearly identified in mechanical tests. For example, the recent work on multiscale DIC application to pantographic structures has revealed additional relative shear deformation modes at the hinges besides providing a detailed kinematic description of fiber bending (Hild et al., 2021). In the present paper, a similar type of multiscale analyses is performed on chiral granular strings subjected to extension. The granular string sample in which the grains were interconnected using a particular "duoskelion" bridge was 3D printed. Two different surface preparations for DIC were applied on the two opposite surfaces of the specimen. The specimen was then subjected to two sequential extension tests, and images acquired at different loading steps for each surface. DIC analyses were then performed at 3 scales: i) macroscale, in which the granular string was treated as a continuum bar; ii) microscale, in which all the detailed microstructural characteristics were fully represented; and iii) mesoscale in which the effort was devoted to obtaining rigid body motions pertinent to granular material systems. Uncertainties associated with the DIC results were analyzed and quantified. The results of DIC at the 3 spatial scales were described with particular attention to the non-standard deformations mechanisms revealed through such analyses.

\section{Sample and Experiment}

\subsection{Sample Preparation}

The prototypical granular string made of 11 grains connected via the conceived connection was rendered using the CAD software SolidWorks (Dassault Systems SolidWorks Corporation, Waltham, MA, USA). The 11-grain string is terminated at both ends with a flat extension designed for gripping the specimen in the testing machine. The dimensions of the features comprising the 1D granular string are given in Figure 1 for the specimen discussed in this paper. The out-of-plane thickness of the granular string was specified to be $4 \mathrm{~mm}$ so as to prevent warpage of the sample in the fabrication process, while also admitting a 2D planar analysis of deformations (Figure 2(a)). The CAD model was fabricated using a "Durable Resin" monomer in the Form 3 3D printer (FormLabs, USA). The Form 3 3D printer is based upon Low Force Stereolithography (LFS) ${ }^{\mathrm{TM}}$, which 
photo-polymerizes the resin into the desired shape. The $X Y$ resolution and layer thickness was $\approx 50 \mu \mathrm{m}$ such that sub-millimeter features were achieved with good precision.

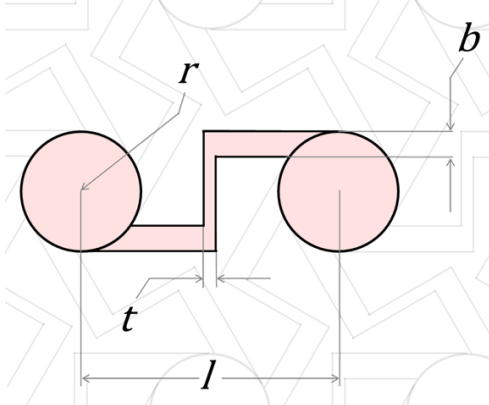

\begin{tabular}{|c|c|}
\hline \hline Geometrical parameter & Dimension $(\mathrm{mm})$ \\
\hline $\mathrm{l}$ & $10.5 \pm 0.1$ \\
\hline $\mathrm{r}$ & $2.5 \pm 0.1$ \\
\hline $\mathrm{t}$ & $0.6 \pm 0.1$ \\
\hline $\mathrm{b}$ & $1.0 \pm 0.1$ \\
\hline
\end{tabular}

Figure 1: Geometrical parameters corresponding to the proposed grain-pair interaction model

\subsection{Experiment}

The fabricated granular string was tested under extension in an ElectroForce 3200 (TA Instruments) mechanical testing machine equipped with a load cell of capacity $\pm 450 \mathrm{~N}$, a measurement uncertainty of $0.1 \%$ and precision of $0.001 \mathrm{~N}$, and a displacement transducer with a range $\pm 6.5 \mathrm{~mm}$, a measurement uncertainty of $0.1 \%$ and precision $0.001 \mathrm{~mm}$. Figure 2(b) shows the experimental setup indicating the clamped boundary conditions, such that on the top grain of the string a vertical displacement is applied while the lateral displacement and rotations are constrained, and on the bottom grain both the vertical and lateral displacements as well as the rotations are constrained. The granular string was stretched at a rate of $0.2 \mathrm{~mm} / \mathrm{s}$ up to a total extension of $10 \mathrm{~mm}$.

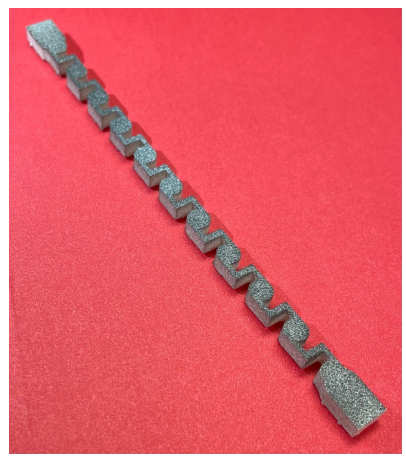

(a)

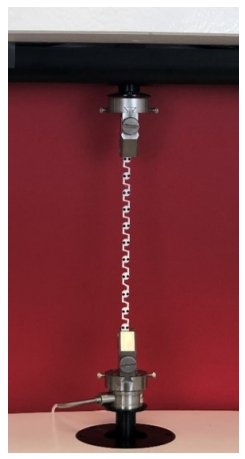

(b)

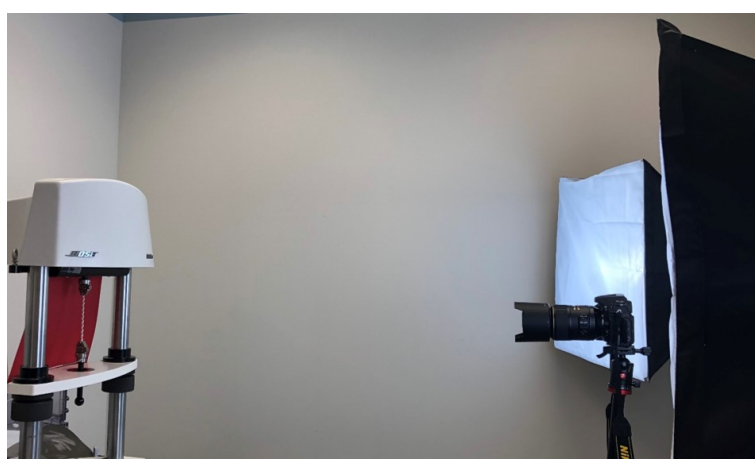

(c)

Figure 2: (a) Speckled sample surface. Experimental (b) and picture acquisition (c) setup 


\section{Table 1}

DIC hardware parameters

\begin{tabular}{|l|l|}
\hline \hline Camera & NIKON D300 \\
Definition & $4288 \times 2848$ pixels (RGB image) \\
Gray Levels amplitude & 8 bits \\
Lens & AF-S VR Micro-Nikkor 105mm f/2.8G ED \\
Aperture & $f / 4.5$ \\
Field of view & $111 \times 74 \mathrm{~mm}^{2}$ \\
Image scale & $60 \mu \mathrm{m} / \mathrm{px}($ B \&W image) \\
Stand-off distance & $\approx 90 \mathrm{~cm}$ \\
Image acquisition rate & $1 / 5-1 / 3$ fps \\
Exposure time & $20 \mathrm{~ms}$ \\
Patterning technique & Sprayed black paint or black dots \\
Pattern feature size & 3 px (speckle pattern) or 31 px (dots) \\
\hline \hline
\end{tabular}

Table 1 lists the hardware parameters of the optical setup. To facilitate data extraction from images, a white background was used in the case of dotted pattern, while a red background was adopted in the case of speckle pattern. For the purpose of image acquisition in both cases, soft boxes were used to generate diffuse lighting (Figure 2(c)).

The extension experiment was repeated twice for the same specimen to acquire images for the two surface patterns. In the first case, two dots were manually drawn on each grain surface (Appendix A). In the second case, a speckle pattern was spray painted upon the surface. While the dotted pattern is a legacy approach to determine the rigid body motion of the grains (Appendix A), the speckle pattern was expected to provide a richer outcome of kinematics at various spatial scales, and detailed deformation behavior of the connections. Figure 3 gives the initial (i.e., undeformed) configuration and all deformed configurations under step-wise extension of the speckled surface. 

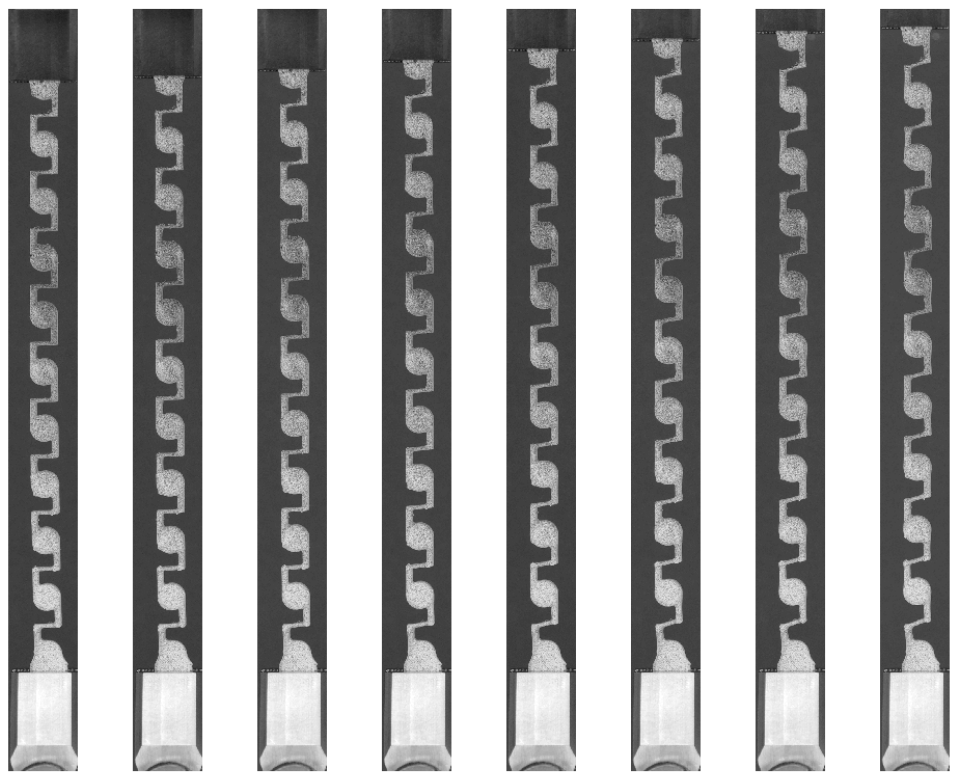

Figure 3: Cropped gray level images of the extensional test with speckled grains

\section{Multiscale DIC Strategies}

With the aim of illustrating the role of micromechanisms, and power of DIC in extracting the details of the deformation behavior, the elongation experiment was analyzed at multiple spatial scales using a suite of different kinematic assumptions. To this end, the granular string may be represented in 3 different ways: i) as a continuous bar devoid of structure when the observation is at a coarse or the macroscale; ii) as a structured bar in which the geometrical features, such as beamlike elements connecting bulky circular grains, are observable, defined here as the microscale; and iii) as a set of rigid interconnected grains whose deformation can be represented by following the grain motions, defined as the mesoscale.

\subsection{Kinematic Bases}

For macroscale analyses, the grain-string structure is taken as a continuum bar of homogeneous cross-section, such that no distinction was made between the solid phase and surrounding air. The macroscale assumption was deemed to represent a very small finite volume element of a large body analyzed at a scale in which the grains were indistinguishable. Finite element discretizations of the 
assumed bar were considered with 3-noded traingular (T3) elements. The only requirement was that the structured meshes encompass the whole solid phase (Figures 5 and 16). Modeling of the behavior of granular materials that emerges at the macroscale are known to be challenging due to the effects of micromechanisms. An analysis at the macroscale without direct representation of the microstructure could aid in revealing these emergent effects. Such results could be useful for evaluation of macroscopic continuum models developed to describe the deformation of such granular materials (Rechenmacher, 2006; Vardoulakis, 2019; Larsson et al., 2020). An alternative application, and one that is particularly useful for the multiscalar efforts described here, is to utilize the macroscale result to initialize more refined DIC analyses with the measured displacement field at the macroscale. Such strategy was used herein and enabled the number of iterations to be significantly lowered for the microscale and mesoscale analyses.

Discrete models of granular materials have been formulated in terms of rigid body motions of the grains given that the deformations are often localized in very small regions where the grains connect with each other (see, for example, the widely used discrete element models (Cundall and Strack, 1979; Jean and Moreau, 1987; Holtzman et al., 2010)). Irrespective of the deformation location, the discrete view that considers grain-displacements and grain-rotation about their barycenters, presents an effective model for describing the collective deformation of large collections of deformable grains (Turco et al., 2019). Therefore, the determination of the grain motions is of wide interest both from the viewpoint of validation of discrete models as well as for the development of plausible grain-interactions relationships relevant to these models as well as those that aim to link grain-scale to macroscale continua (Nejadsadeghi and Misra, 2020). From the discrete model (defined here as the mesoscale) viewpoint, the deformation of the string can be represented by following the motions of the set of grains.

In this work, mesoscale analyses were performed in two ways. First, mesoscale analyses (i.e., at the scale of each grain) were conducted with 3 degrees of freedom (DOFs) per grain (i.e., in-plane translations of the grain center, and corresponding rigid body rotation) for both surface prepa- 
rations. In all these cases, circular regions of interest (ROIs) were considered. Second, for the speckled surface, 6 DOFs per grain were also analyzed (i.e., in-plane translations of the grain center, and corresponding mean in-plane deformation gradient). The 6 DOFs analysis was feasible for the speckled surface due to the availability of information over the whole grain surface as opposed to the case of dotted surface. Such an analysis can aid in validating the mesoscale treatment of the granular string using a discrete model by evaluating the state of deformation within the grain.

At the microscale of the granular string, the deformation was analyzed for the detailed geometrical structure for the speckled surface. A preliminary step was required to construct meshes that were consistent with the underlying mesostructure (Figures 4(left) and 18(right)). To this end, a so-called backtracking procedure was utilized (dell’Isola et al., 2019; Hild et al., 2021). From the knowledge of the nominal geometry, a mask was created (Figure 4(center)) and a mesh was generated using Gmsh (Geuzaine and Remacle, 2009). The two images were then registered via regularized DIC with an auxiliary mesh corresponding to the first discretization at the macroscale (Figure 5(left)). At the end of the procedure, the mesh was backtracked by using the measured displacement field (Figure 4(right)). This operation was also applied to the center of each grain that was initially determined in the nominal configuration. For the dotted surface, discrete analyses were performed in which 4 T3 elements were considered with one node for each element coincident with the dot center (Figure 18). Microscale analyses are particularly useful for understanding the behavior of grain interconnections. Needless to say, the microscale analysis is limited by the resolution of the image and may not reveal all the details of the interconnection deformation. However, the intent in this particular work was not resolve how every element of the interconnections deformed but to reveal which aspect of the interconnections underwent the deformation. The aim of this analysis was to understand how the geometry of the interconnections could be modified to modulate the behavior of the overall system. 

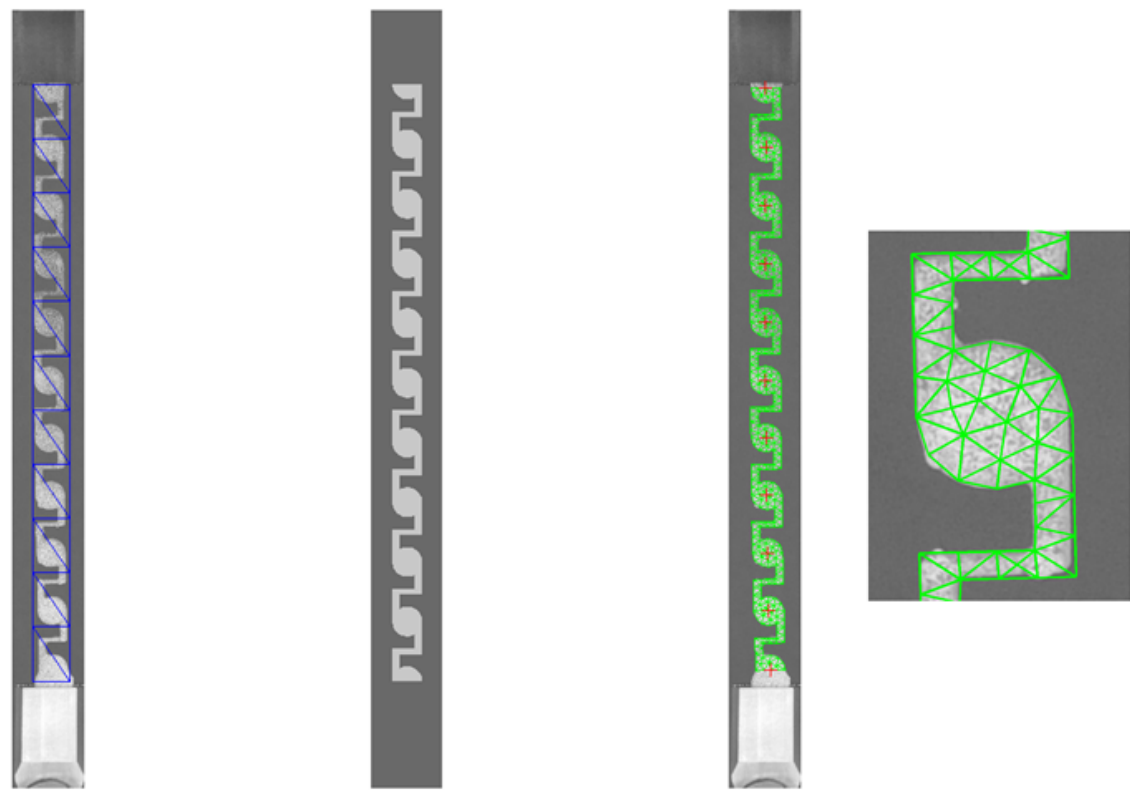

Figure 4: Backtracked mesh for microscale DIC of the speckled surface. The image of the reference configuration (left) was registered with that of the nominal configuration (middle) using the auxiliary mesh shown in blue. The backtracked mesh was laid over the reference image (right). Mesh details are shown for one of the grains. The grain centers (red crosses) are also depicted

For the results described in this paper, the DIC analyses were performed using the Correli 3.0 framework (Leclerc et al., 2015) in which Hencky-elastic regularization was implemented (Table 2). The DIC framework followed herein is summarized in Appendix B. 


\section{Table 2}

DIC analysis parameters

\begin{tabular}{|l|l|}
\hline \hline DIC software & Correli 3.0 (Leclerc et al., 2015) \\
Image filtering & none \\
\hline Element length (mean) & see text \\
Shape functions & linear (T3) \\
Mesh & see Figures 5, 16, 4 and 18 \\
Matching criterion & penalized sum of squared differences \\
Regularization length & 100 px \\
\hline Grain radius & 50 px \\
Shape functions & 3 or 6 (see text) \\
Matching criterion & sum of squared differences \\
\hline Interpolant & cubic \\
Displacement noise-floor & see Table 3 \\
\hline \hline
\end{tabular}

\subsection{Uncertainty Quantification}

The displacement noise-floor is an important information for assessing the performance of the selected DIC routine (iDICs et al., 2018; ASD-STAN prEN 4861 P1, 2018). In the present case, there was only one image of the reference configuration. Fortunately, the sample structure covered a considerably small region in comparison to the full definition of the images. Consequently, there were a large number of pixels in the background that did not cover the structures of interest. Assuming that the camera remained stationary during the experiment as per the experimental protocol, it was possible to crop a large ROI of the background from all the images. All independent ROI differences were computed and acquisition noise was estimated from the average variance of each pixel of the ROI. The square root of the variance is then equal to $\sqrt{2} \sigma$, where $\sigma$ denotes the standard deviation of acquisition noise corrupting each image. For the two experiments reported in this work, the lighting conditions and backgrounds were not identical. As expected, the stan- 
dard deviations $\sigma$ were different as well, namely, for the dotted surface $\sqrt{2} \sigma=1.2$ gray level, and $\sqrt{2} \sigma=2.4$ gray levels for the speckle pattern.

Using the above estimate of acquisition noise, uncertainty quantification was performed by generating a series of 100 pictures in which Gaussian white noise was added with a standard deviation of $\sqrt{2} \sigma$ to the image of the reference configuration. DIC analyses were then run on these image series. The temporal standard deviation was then evaluated for each DOF $v_{i}$ considered in the DIC analysis (Appendix B). For finite element discretizations, the mean variance was assessed per direction, and then its square root was obtained to assess the standard displacement uncertainty.

Table 3 gathers the results of this uncertainty quantification. Since one of the surfaces had only dots with no speckle pattern, the regularization length was increased to enable for a good convergence of DIC analyses. In the present case, $\ell_{\text {reg }}=100 \mathrm{px}$ for all meshes. Because the regularization length was greater than the element sizes considered herein, the uncertainties were controlled by the former. No dependence on mesh size was observed for both macroscale and microscale analyses. In addition, very low levels of uncertainties were achieved. In contrast, the discrete analyses around each black dot concerned very small areas for which the standard displacement uncertainty increased in comparison to continuous meshes. Last, for the analyses at the grain level with 3 or 6 DOFs led to displacement uncertainties of the same order of magnitude than those observed with continuous meshes. The standard uncertainty levels for the last degrees of freedom referring to the rotation or the deformation gradient were rather small. This is due to the fact that the grain radius was equal to $50 \mathrm{px}$. 


\section{Table 3}

Standard uncertainties of each DOF for the analyses at three different scales and for the two surface preparations. See text for the description of the kinematics associated with 3 and 6 DOFs

\begin{tabular}{|l|c|c|}
\hline \hline DIC analysis & Speckle & Dots \\
\hline Macroscopic scale & $0.004 \mathrm{px} \mid 0.004 \mathrm{px}$ & $0.004 \mathrm{px} \mid 0.003 \mathrm{px}$ \\
Microscopic scale & $0.007 \mathrm{px} \mid 0.007 \mathrm{px}$ & $0.010 \mathrm{px} \mid 0.008 \mathrm{px}$ \\
Discrete & -- & $0.013 \mathrm{px} \mid 0.013 \mathrm{px}$ \\
3 DOFs & $0.003 \mathrm{px}|0.003 \mathrm{px}| 9 \times 10^{-5}$ & $0.002 \mathrm{px}|0.002 \mathrm{px}| 6 \times 10^{-5}$ \\
6 DOFS & $0.003 \mathrm{px}|0.003 \mathrm{px}| 1.1 \times 10^{-4}$ & -- \\
\hline
\end{tabular}

\section{Probing the Three Different Kinematic Descriptions}

The gray level residuals are first analyzed as a way of probing the three kinematic bases before discussing the findings from the DIC results. The applicability of the proposed multiscalar registrations is evaluated by investigating the evolution of gray level residuals as the loading progresses for each of the three kinematic bases. The gray level residuals indicate the suitability of each basis.

\subsection{Macroscale analyses}

Figure 5 shows the three meshes considered for the macroscale analyses. To characterize the mesh density, the element size was defined as the square root of the mean element surface. For the speckled surface (Figure 5), the three element sizes were 79, 39, and 22 px, respectively. These meshes were used to perform a convergence analysis by running DIC analyses with each mesh on the image series. Two sets of results are reported. First, so called incremental DIC was conducted in which the reference configuration of a new calculation corresponds to the previous deformed configuration. This is possible since the mesh was gradually deformed according to the measured incremental displacement. Such analysis enables very large displacement amplitudes to be measured, and the Lagrangian displacements are obtained by adding all incremental contributions. However, it leads to an accumulation of DIC measurement errors. Once this first analysis was 

both DIC routes.

Figure 5: Three meshes used for the DIC analyses at the macroscale of the speckled surface

completed, a second one (referred to as direct) was carried out in which the reference picture was always the initial one, and the initial guess for any time step was that provided by the incremental procedure. In the following, RMS residuals $\varrho_{c}$ (see Appendix B) are analyzed for any mesh and

Figure 6 shows the RMS residuals for the 7 analyzed pictures of the speckled surface with the three different meshes. For the coarsest mesh (Figure 6(a)), no significant gain is observed when direct and incremental analyses were performed. A small decrease is observed when finer meshes were selected (Figure 6(b-c)). As the meshes were refined, the RMS residuals decreased a bit. However, they could not be significantly lowered. This is due to the fact that the regularization length was larger than the element size of any of the considered meshes. Last, it is worth noting that the RMS residuals are significantly larger (i.e., between 2 and 7 times) than the level associated with acquisition noise (i.e., 2.4 gray levels in the present case). Further, they degrade as the applied displacement amplitude increased. This trend is not a shortcoming of the registration procedure itself but is rather related to the fact that the selected kinematic basis was no longer able to fully 
capture the details of the deformations at the microstructural level.

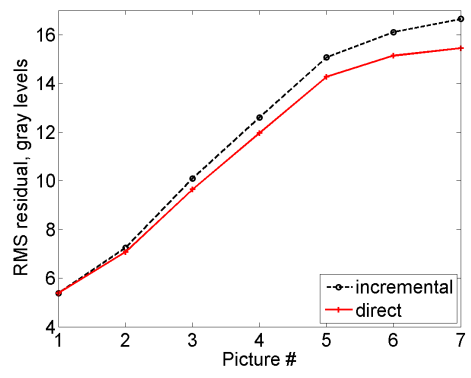

(a)

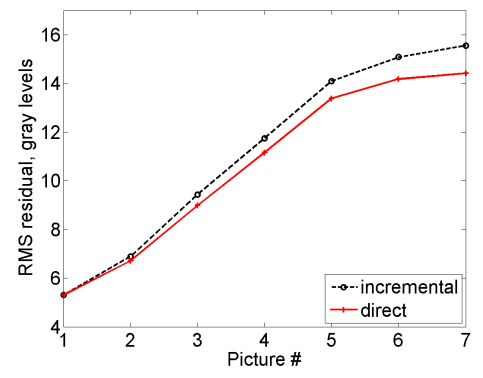

(b)

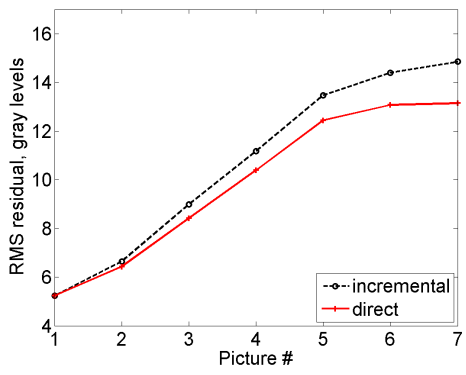

(c)

Figure 6: RMS gray level residuals for the three meshes of the speckled surface (Figure 5) and the two DIC routes

From the convergence analysis, it is concluded that any of the selected meshes is able to give acceptable results for initialization purposes. No significant gain was observed when refining the discretization because of the large regularization and the fact that the geometry was not adapted to the underlying microstructure. This observation also applies to the results obtained with the dotted surface (Figure 17).

\subsection{Mesoscale analyses}

The following analyses were performed to extract the kinematic information at the grain-scale. Circular ROIs were considered for each grain and DIC analyses were run independently. For each grain, 3 DOFs were considered (i.e., 2 rigid body translations and 1 rigid body rotation). Figure 7(a) shows the change of RMS residuals for each grain and each image of the considered series. The RMS residuals vary from 1 to less than 2 times the level associated with acquisition noise, which is very low. These results prove that the selected kinematic basis is able to capture very well the underlying motions of grains. 

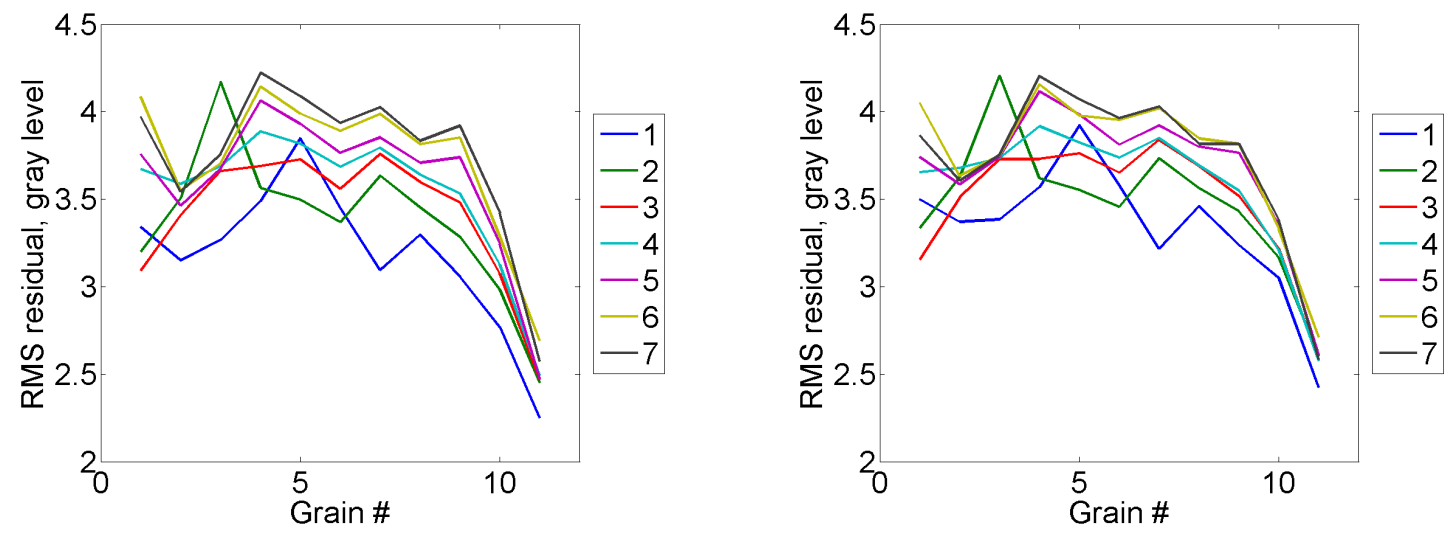

(a)

Figure 7: RMS gray level residuals for the DIC analyses with 3 DOFs (a) and six DOFs (b) per grain of the speckled surface (each line corresponds to one picture of the series)

To verify the assumption that the bulk of the grain suffered indistinguishable deformation, an additional analysis was performed by allowing for uniform strains at the grain-scale (i.e., defining the kinematics using 6 DOFs instead of 3 for the rigid body motions). The RMS residuals for the 3DOF solution (Figure 7(a)) and with 6 DOFs (Figure 7(b)) show insignificant differences, proving that the analyses with 3 DOFs were sufficient to capture the overall motions of each grain. It is concluded that the bulk of the grain does not experience strain and the grain movement is governed by the deformation at the connections between grain-pairs.

\subsection{Microscale analyses}

In the present case, the mean element size was equal to $16 \mathrm{px}$. Contrary to the macroscale analyses, the RMS residuals did not vary significantly with the applied displacement (Figure 8). Their average was of the order of 2.3 times the level due to acquisition noise. It is concluded that the kinematics was very well captured by such analyses. If deformation details were to be sought, then the mesh should be further refined (see Section 5.3), which is possible thanks to the regularization strategy used herein. 


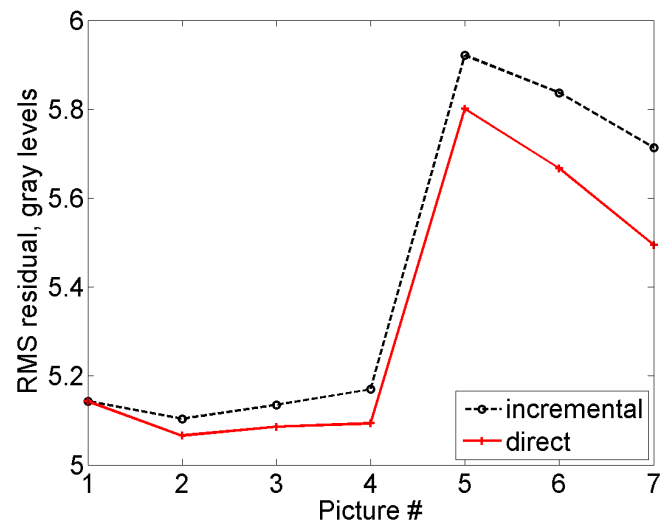

(a)

Figure 8: RMS gray level residuals for the DIC analyses at the microscale of the speckled surface

This first series of results show that the macroscopic kinematics could be captured. However, the degradation of the gray level residuals indicate that such description is not able to fully account for local details of the deformation mechanisms of the granular string. For the grains themselves, the hypothesis of rigid body motions was validated for any applied displacement amplitude. Last, the analysis at the microscale was shown to be feasible thanks to the mesh that was tailored to the underlying morphology of the granular string and its interconnections, in addition to the regularization scheme based on Hencky elasticity.

\section{Results for the Speckled Surface}

The DIC results obtained from the analyses at different scales are now further commented for the case of speckled surface. The discussion here focuses upon the deformation mechanisms revealed through the multiscalar analysis. Similar results are obtained for the case of dotted surface and are discussed in Appendix A.

\subsection{Macroscale Analysis}

Figure 9 gives the results of the direct analysis for the finest discretization and the final image of the extension test (i.e., the image at maximum applied displacement). Given that the maximum displacement amplitude (i.e., $180 \mathrm{px}$ ) was quite large, and the total number of images was few 
(i.e., 7 in the present case), the incremental analysis was the only one able to capture such large displacement increments, and was very useful to initialize subsequent direct DIC analyses.

The underlying geometry of the granular string expresses itself in the transverse displacement field (Figure 9(left)) in which a fluctuating pattern of positive and negative transverse displacements is observed. On the other hand, the longitudinal displacement field (Figure 9(middle)) shows a nominally monotonically varying trend. To explicate any fluctuating pattern in the longitudinal field, the mean strain component was deducted from the measured field. Displacement fluctuations are now observed in Figure 9(right), whose overall pattern is similar to that observed for the transverse component. These fluctuations (or deviations) from the erstwhile linear field is a characteristics of granular material, which was first experimentally observed in (Misra and Jiang, 1997), and are generally attributed to the randomness of the granular structure. It is remarkable, therefore, to observe similar fluctuations in this rather simple grain-string with regularly spaced grains and nominally uniform interconnections whose genesis could be possibly traced to mechanical features of grain-pair interactions. It is also interesting to note that the levels of fluctuations are close for both displacement components.

In Figure 9(rightmost), the gray level residual map is reported for the last analyzed image. It is worth noting that the grain areas do not have clear residuals. However, the connecting beams are clearly visible, which means that their kinematics was not properly captured. These two points show that in the macroscale analysis, the overall deformations are correctly captured. However, the details of the connecting parts of the grains were not. In the absence of complete knowledge of microstructure and micromechanics (hence a possibility for microscale DIC analyses), macroscale DIC provides significant clues, albeit with an incomplete description, regarding the effect of the underlying micro-mechano-morphology. For the analyzed grain-string, tantalizing transverse displacement fields and longitudinal fluctuations were extracted, although specific details may only be revealed in microscale or mesoscale analyses as shown in the subsequent results. 

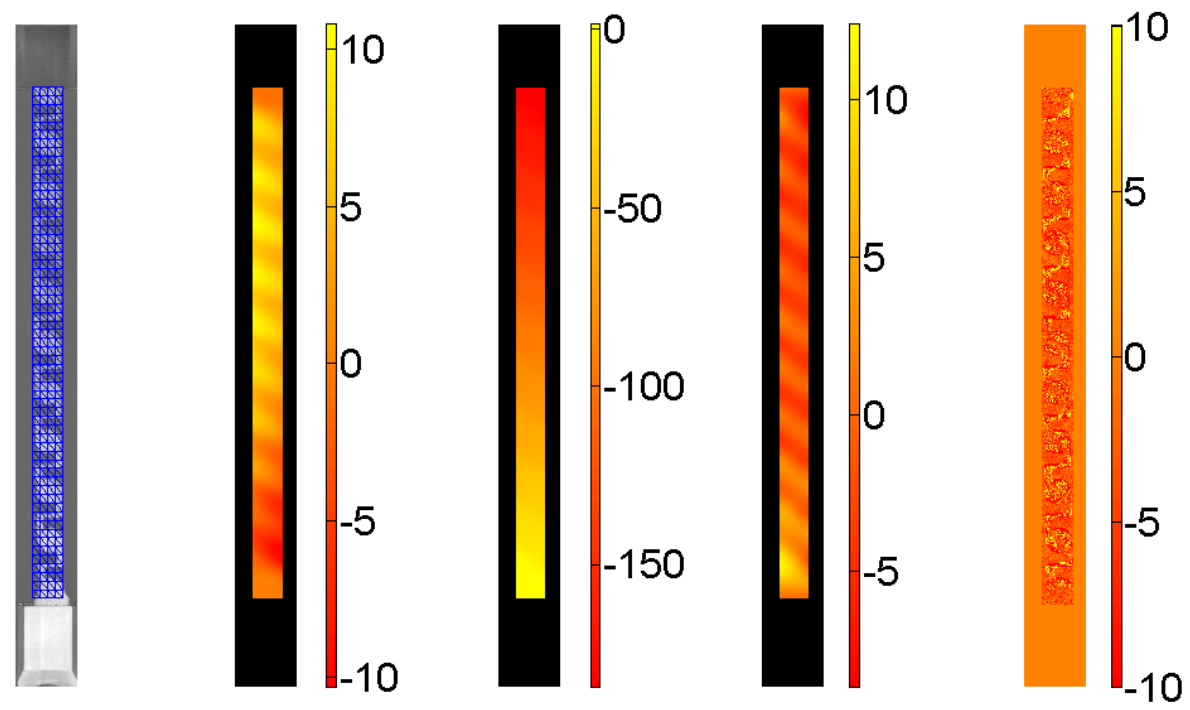

Figure 9: Mesh laid over the reference picture for the macroscale analysis of the speckled surface (leftmost). Transverse displacement (expressed in px) field for the last analyzed picture (left). Corresponding longitudinal displacement (expressed in px) field (middle) and displacement fluctuations once the mean strain contribution was taken out (right). Gray level residual field (rightmost)

\subsection{Mesoscale Analysis}

The mesoscale analyses dealt rigid body motions of the grains, thus the kinematics was reduced to 3 DOFs for each grain (Section 4.2). Figure 10 shows the change of the grain-displacement components and grain-rotation about their barycenters, where different curves represent different overall extensions of the grain-string. Since the boundary grains were held tightly in the grips of the loading frame, the transverse displacement and the rotations of the boundary grains were zero. It is interesting to note that the longitudinal displacement was predominantly linear with respect to the grain number (also with grain-location since they are equally spaced). This trend was already observed in the macroscale results (Figure 9). To confirm this observation, the mean strain contribution was taken out to report the remaining fluctuations (Figure 10(c)). Interestingly, such fluctuations consistently increased with the applied load (i.e., their pattern was set early on 
and is a further confirmation that it is the signature of the underlying mesostructure).

The transverse displacement, on the other hand, has a sinusoidal-like pattern that formed as the applied displacement increased. Similarly, grain-rotations (due to the asymmetry of the underlying structure of grain interconnections, or in an alternative interpretation the grain-pair mechanical interactions, with respect to the loading direction) increased with the applied overall extension. It is also noteworthy that the grain rotations are coherent in the counter-clockwise direction, that is, all the grain rotations are in the same orientation as opposed to gear-like opposite rotations, which are also observed in granular systems (Kuhn and Bagi, 2004; Harrington et al., 2014; Wang et al., 2021). It is further observed that the system supports grain-rotation gradients such that energy is stored in grain-pair relative rotations. 


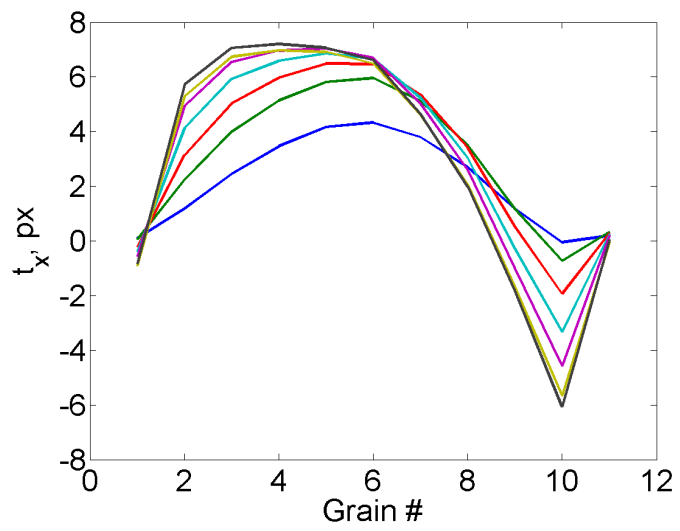

(a)

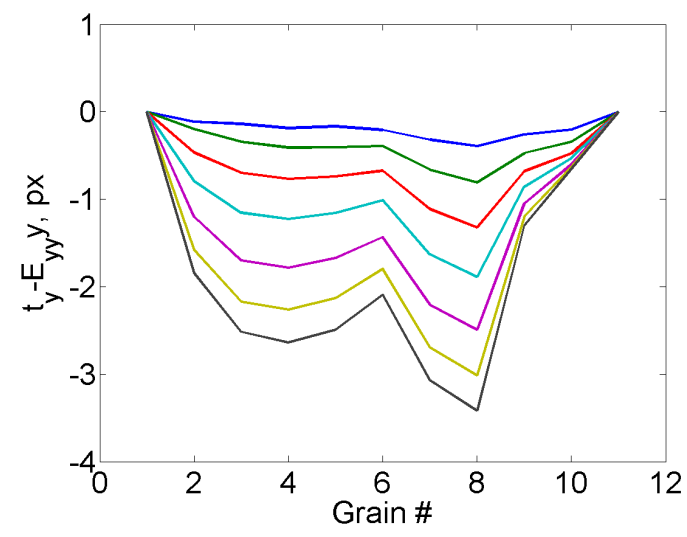

(c)

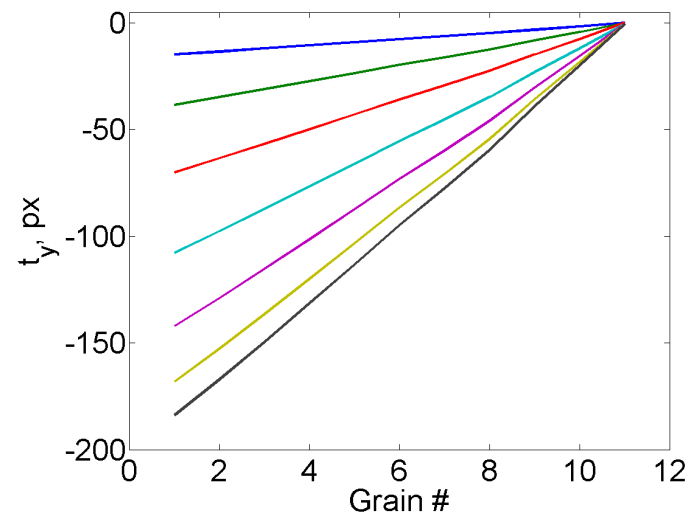

(b)

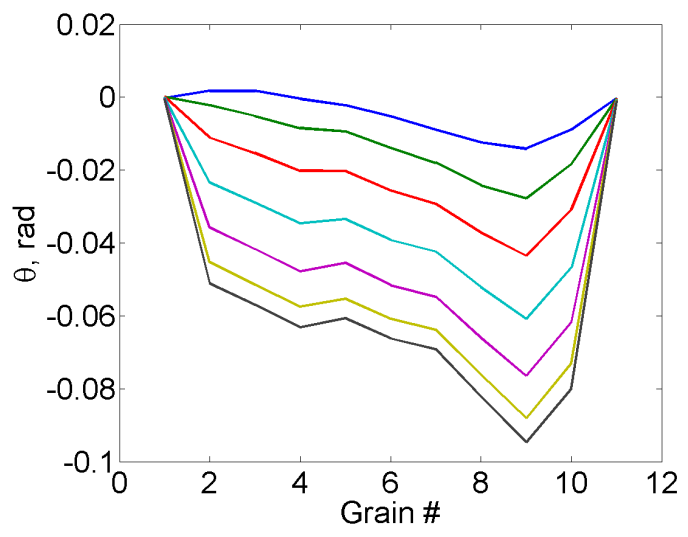

(d)

Figure 10: Change of the transverse translation (a), longitudinal translation (b), fluctuations of the longitudinal translation (c), and rotation (d) of the eleven grains for all analyzed images of the speckled surface (for line color, refer to Figure 7)

To confirm the granular nature of the deformation behavior of the granular-string, the findings from the analysis with 6 DOFs per grain are shown in Figure 11. The rigid body translations are virtually identical to those observed in Figure 10. The normal components of the deformation gradient tensor (i.e., $F_{x x}$ and $F_{y y}$ ) have amplitudes that are less than $1 \%$ with respect to unity (i.e., very low levels of normal strains). Conversely, the off-diagonal components are virtually identical in absolute value indicating very small shear strains and mainly rigid body rotations. These observations also explain why the gray level residuals were very close for the analyses with 3 and 6 DOFs 

per grain (Figure 7).

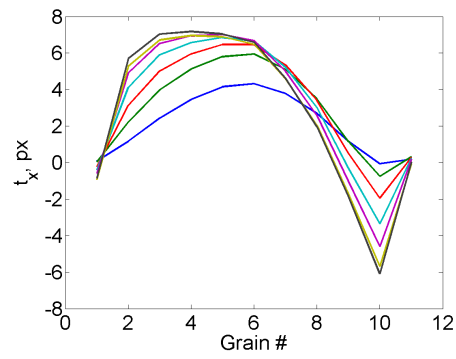

(a)

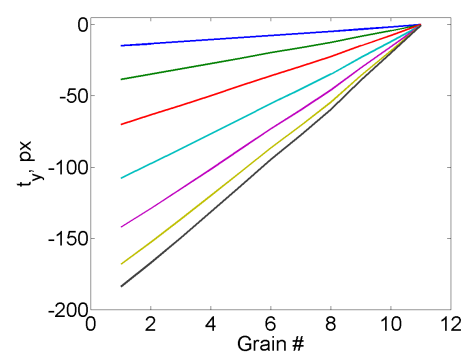

(d)

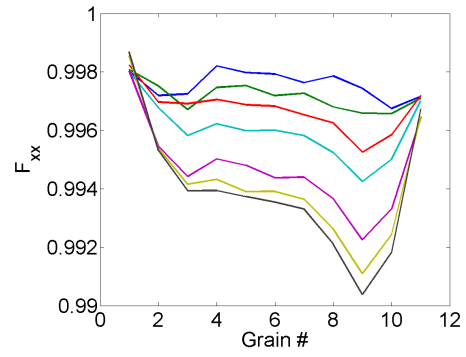

(b)

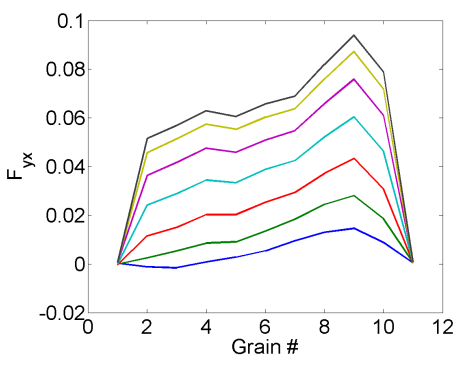

(e)

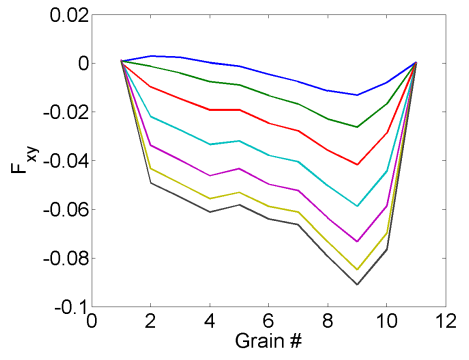

(c)

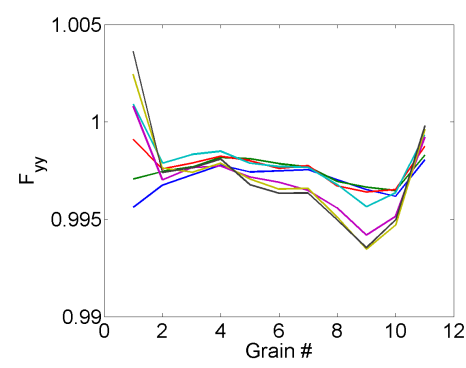

(f)

Figure 11: Evolution of the six degrees of freedom of the eleven grains for all analyzed images of the speckled surface. Each curve corresponds to a different-level of applied overall extension (for line color, refer to Figure 7)

\subsection{Microscale Analysis}

For the grain-string, the details of the microstructure, particularly with respect to their interconnections, are known. Thus, microscale DIC analyses were performed to better capture the local details of deformations (Figure 12). In that case, the mesh was tailored to fit the initial geometry with the backtracking procedure (Figure 12(leftmost)). The transverse displacements are now clearly revealed (Figure 12(left)) in comparison to the macroscale result (Figure 9(left)). The transverse motions were strongly influenced by the underlying geometry of the granular string. The longitudinal displacements (Figure 12(right)) seem to remain close to their macroscale estimates (Figure 9(middle). However, once the mean strain component was subtracted, significant fluctuations are observed, which are primarily concentrated in the grain interconnections further 
confirming that their likely origin is due to the features of grain-pair mechanical interactions. Needless to say, the results at the microscale are more detailed as well as reliable as indicated by the gray level residual map that no longer shows high levels for the connecting parts and remains low in the grains as well.
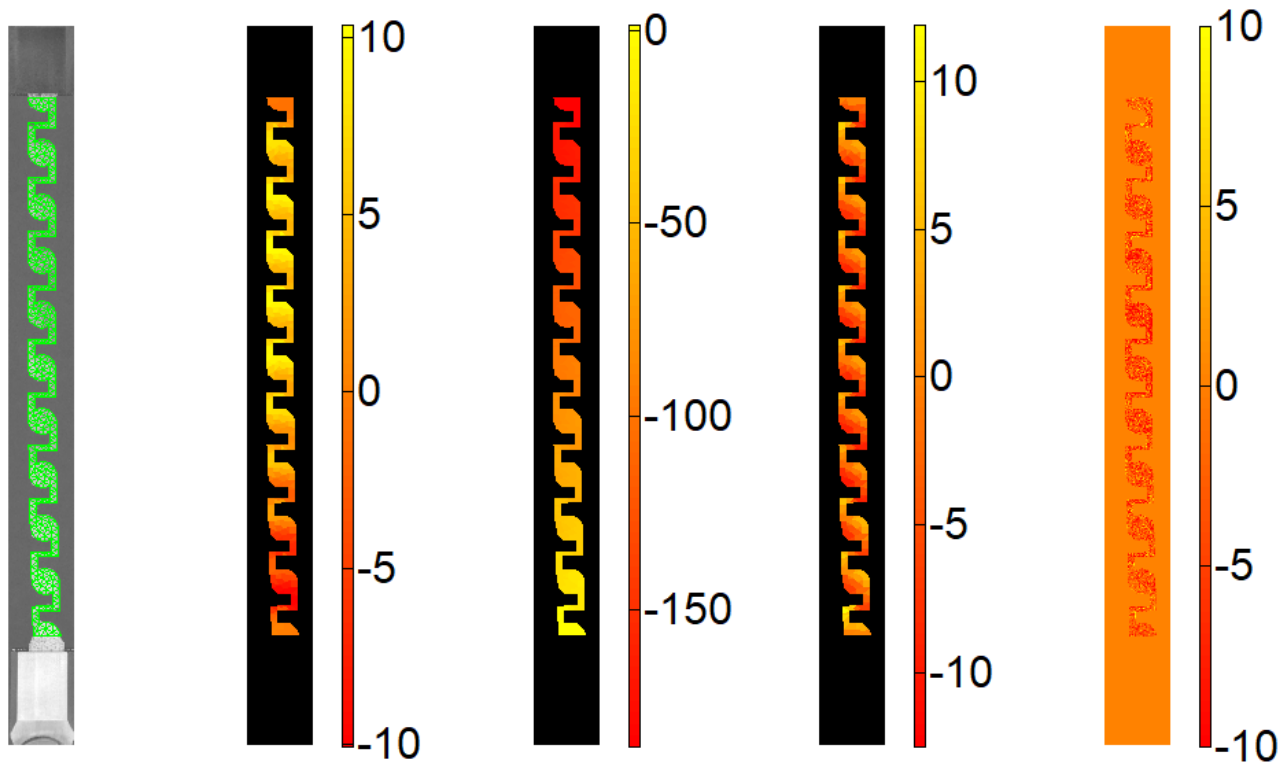

Figure 12: Mesh laid over the reference picture for the microscale analysis of the speckled surface (leftmost). Transverse displacement (expressed in px) field for the last analyzed picture (left). Corresponding longitudinal displacement (expressed in px) field (middle) and displacement fluctuations (right) once the mean strain contribution was taken out. Gray level residual field (rightmost)

From this first set of results at the microscale arise two additional questions. First, given the fineness of the interconnections, regularized DIC was required. There is still a choice to be made when the regularization length $\ell_{\text {reg }}$ is greater than the element (as was the case herein). Different analyses were run starting with the largest length (i.e., 800 px). The next DIC run was carried out by dividing the regularization length by 2 and initializing the calculations with the results obtained with the previous regularization length. This relaxation procedure (Tomičević et al., 2013) was carried out down to a regularization length of 50 px. Figure 13 shows the change of the equilibrium residual $\varrho_{m}$ as a function of the registration residual $\varrho_{c}$ for the 7 analyzed pictures. As more weight 
was put on the penalty term (i.e., $\ell_{\text {reg }}$ was increased), the equilibrium residuals were lowered, and the RMS gray level residuals started to increase. This is a typical feature of such regularized schemes (Hansen, 2010).

Further, it is interesting to note that for the first two pictures, the gray levels residuals remained essentially constant for any selected regularization length. This observation shows that the underlying hypothesis of Hencky elasticity was valid. Conversely, from the third picture on, this trend is non longer observed, and the gray level residuals increased more significantly as the regularization length was increased, which indicates a model error. This effect means that nonlinear phenomena occurred and were not accounted for by Hencky elasticity. As a consequence, the chosen regularization length should not be too high. In the present case, a length of $100 \mathrm{vx}$ was selected, which was a good compromise between the two cost functions, particularly for revealing the deformation trends.

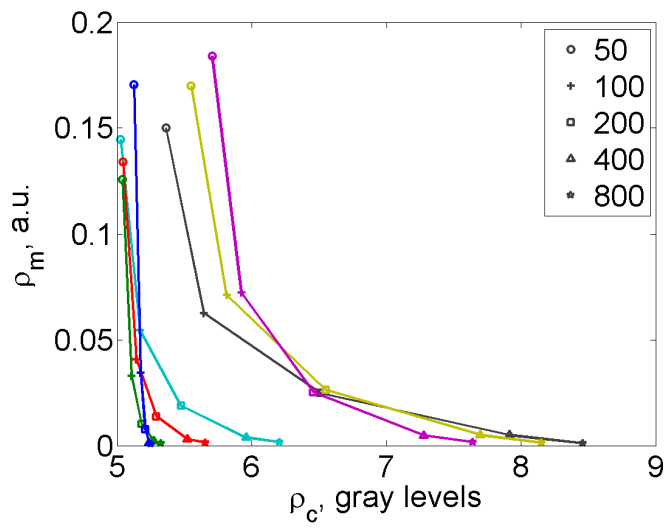

Figure 13: Equilibrium residual $\varrho_{m}$ vs. RMS gray level residuals $\varrho_{c}$ for different regularization lengths $\ell_{\text {reg }}$ (expressed in $\mathrm{vx}$ ) and all analyzed pictures (for line color, refer to Figure 8)

The second question is related to the deformation mechanisms of the interconnections that led to non classical results at the meso- and macroscales. For this type of investigation, the $16 \mathrm{px}$ discretization was too coarse as there were at most 2 elements in the thickness of the interconnecting beams (Figure 4(right)). A very fine mesh was then constructed with a mean element size of 3 px, which allowed for a least six elements in the thickness of each beam. In terms of overall 
displacement fields, there were not many differences given their very high amplitudes. However, using this very fine mesh more local strain details could be better captured. In the following results, the Green-Lagrange strains are reported. They were assessed from the deformation gradient tensor, which was obtained from the exact derivation of the T3 shape functions (i.e., they are uniform over each element).

The transverse Green-Lagrange strain field is shown in Figure 13(leftmost) for the last analyzed picture. Very low strain levels are observed in the vertical beams and the grains. The horizontal beams of each interconnections undergo transverse strain that are consistent with flexure. This result was expected from the mesoscale analyses which show the transverse motion and rotation of grains. Figure 13(left) shows the corresponding longitudinal strain field. These strain fields are consistent with the flexure of the vertical beams, whereas the horizontal beams mostly elongated. In terms of shear strains (Figure 13(right)), they were more important in the horizontal beams than in the vertical ones. In the grains, all strain components were very low in magnitude, which further validates the hypothesis of rigid body motions. 

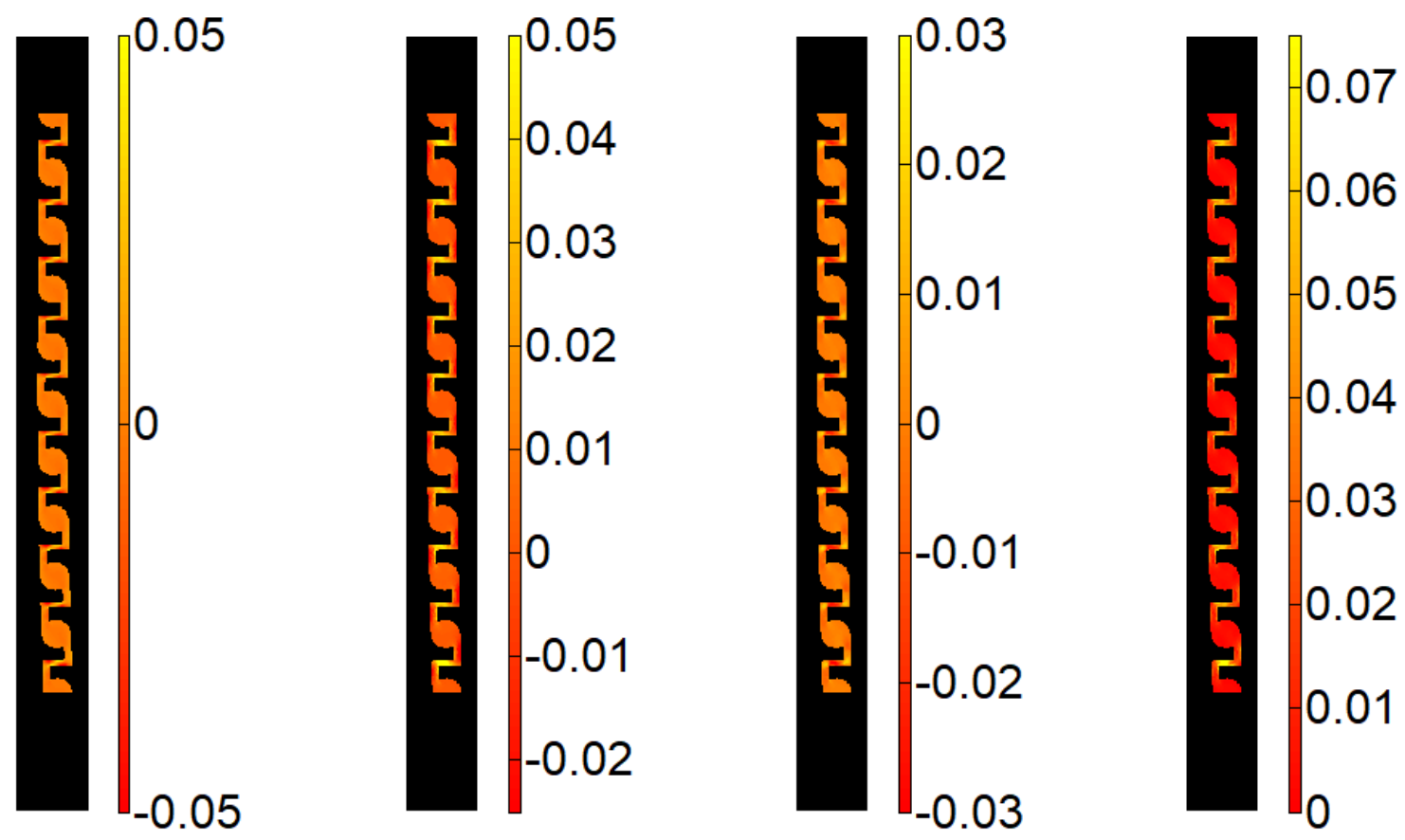

Figure 14: Transverse strain field for the last analyzed picture (leftmost). Corresponding longitudinal (left) and shear (right) strain fields for the last analyzed picture. Equivalent elastic strain field (rightmost)

Last, to assess the distribution of strain energy, the equivalent elastic strain field is shown in Figure 13(rightmost). The latter corresponds to the square root of twice the strain energy (computed with a plane stress assumption) divided by the Young's modulus. Most of the elastic energy was stored in the horizontal beams, whose deformation was a combination of extension and flexure. This result shows that the grain-string can be treated as a granular matter composed of nearly rigid elements (or grains) and the elastic strain energy is stored in the deformable mechanisms represented through interconnections or interfaces between grains. Furthermore, the deformation of the grain string is controlled by that of the interconnections, and therefore can be modulated by varying the geometrical attributes of the interconnections.

\section{Conclusion}

The deformation behavior of a chiral granular string was analyzed using DIC devised for three spatial scales, termed here as macro, meso and micro. The registration residuals of the kinematic 
bases probed at the different scales showed very low levels at two of the chosen scales (namely, micro- and meso-scales) indicating that the deformation mechanisms were properly captured. Although the macroscale description was not as trustworthy, it, nevertheless, provided the necessary initial estimates that were very useful for analyses at lower scales. This choice was particularly important since the displacement increments between adjacent images were very high.

It is also noteworthy that multiscalar analyses have the advantage of elucidating deformation mechanisms that may not be clearly discernible at the macroscale or whose implication at the macroscale is not clear if the focus is only on the microscale. Remarkably, a physical material with microstructure is more intricate than its mathematical model. It may encompass deformation mechanisms that are usually overlooked when modeling. Multiscale DIC analyses offer points of comparison to the model predictions, with which a validation or a need for a refined kinematic description can be concluded.

For the grain-string analyzed in this work, the performed DIC analyses revealed certain nonstandard deformation mechanisms whose genesis is likely to be the chiral-nature of the mechanical response of grain-pair interconnections. As the granular string was subjected to extension, coherent grain rotations (as opposed to gear-like) and transverse grain displacements were observed which increased in magnitude as the loading progresses. In addition, fluctuations from the linear field in the longitudinal displacements were observed. The quantification of the deformation mechanisms in the interconnections with results utilizing a very fine mesh at the microscale confirm the kinematic bases for mesoscale analysis. Further, these microscale results indicate that the nature and magnitude of the non-standard deformations is controlled by the mechanics of grain-pair interactions, which in turn is governed by the geometrical parameters of the grain interconnections for a given fabricated (meta)material. These aspects will be further explored experimentally and theoretically in forthcoming works. 


\section{Acknowledgements}

This research is supported in part by the United States National Science Foundation grant CMMI-1727433.

\section{Credit authorship statement}

NN performed the experiments, MDA assisted in granular string design, AM conceived the idea, FH devised the DIC methodology and performed the DIC analyses. All authors contributed to the discussion of all aspects of this work. NN, MDA, AM and FH wrote and edited the manuscript.

\section{Compliance with Ethical Standards}

The authors have no conflict of interest to declare.

\section{References}

Alderson, A., Alderson, K., Attard, D., Evans, K., Gatt, R., Grima, J., Miller, W., Ravirala, N., Smith, C., Zied, K., 2010. Elastic constants of 3-, 4and 6-connected chiral and anti-chiral honeycombs subject to uniaxial in-plane loading. Composites Science and Technology 70, 1042-1048. Alibert, J.J., Seppecher, P., deel’Isola, F., 2003. Truss Modular Beams with Deformation Energy Depending on Higher Displacement Gradients. Mathematics and Mechanics of Solids 8, 51-73.

ASD-STAN prEN 4861 P1, 2018. Metrological assessment procedure for kinematic fields measured by digital image correlation. /www.asdstan.org/downloads/asd-stan-pren-4861-p1.

Auger, P., Lavigne, T., Smaniotto, B., Spagnuolo, M., dell'Isola, F., Hild, F., 2021. Poynting Effects in Pantographic Metamaterial Captured via Multiscale DVC. Journal of Strain Analysis for Engineering Design (in press) DOI: 10.1177/0309324720976625.

Barchiesi, E., dell'Isola, F., Bersani, A., Turco, E., 2021. Equilibria determination of elastic articulated duoskelion beams in $2 d$ via a riks-type algorithm. International Journal of Non-Linear Mechanics 128, 103628.

Barchiesi, E., Spagnuolo, M., Placidi, L., 2019. Mechanical metamaterials: a state of the art. Mathematics and Mechanics of Solids 24, $212-234$.

Biswas, R., Poh, L., Shedbale, A., 2020. A micromorphic computational homogenization framework for auxetic tetra-chiral structures. Journal of the Mechanics and Physics of Solids 135, 103801.

Bruno, L., Decuzzi, P., Gentile, F., 2016. Stress distribution retrieval in granular materials: A multi-scale model and digital image correlation measurements. Optics and Lasers in Engineering 76, 17-26.

Chen, Y., Frenzel, T., Guenneau, S., Kadic, M., Wegener, M., 2020. Mapping acoustical activity in 3d chiral mechanical metamaterials onto micropolar continuum elasticity. Journal of the Mechanics and Physics of Solids 137, 103877.

Chen, Y., Liu, X., Hu, G., Sun, Q., Zheng, Q., 2014. Micropolar continuum modelling of bi-dimensional tetrachiral lattice. Proceedings of the Royal Society A470, 20130734.

Claire, D., Hild, F., Roux, S., 2004. A finite element formulation to identify damage fields: The equilibrium gap method. Int. J. Num. Meth. Engng. 61, 189-208. 


\section{Multiscalar DIC Analyses Reveal Non-standard Deformation Mechanisms}

Cundall, P.A., Strack, O., 1979. A discrete numerical model for granular assemblies. Géotechnique 29, 47-65.

De Angelo, M., Placidi, L., Nejadsadeghi, N., Misra, A., 2020. Non-standard Timoshenko beam model for chiral metamaterial: Identification of stiffness parameters. Mechanics Research Communications 103, 103462

dell'Isola, F., Seppecher, P., Spagnuolo, M., Barchiesi, E., Hild, F., Lekszycki, T., Giorgio, I., Placidi, L., Andreaus, U., Cuomo, M., Eugster, S., Pfaff, A., Hoschke, K., Langkemper, R., Turco, E., Sarikaya, R., Misra, A., De Angelo, M., D’Annibale, F., Bouterf, A., Pinelli, X., Misra, A., Desmorat, B., Pawlikowski, M., Dupuy, C., Scerrato, D., Peyre, P., Laudato, M., Manzari, L., Göransson, P., Hesch, C., Hesch, S., Franciosi, P., Dirrenberger, J., Maurin, F., Vangelatos, Z., Grigoropoulos, C., Melissinaki, V., Farsari, M., Muller, W., Abali, E., Liebold, C., Ganzosch, G., Harrison, P., Drobnicki, R., Igumnov, L., Alzahrani, F., Hayat, T., 2019. Advances in Pantographic Structures: Design, Manufacturing, Models, Experiments and Image Analyses. Continuum Mechanics and Thermodynamics 31, 1231-1282.

dell'Isola, F., Barchiesi, E., Misra, A., 2020. Naïve Model Theory: its applications to the Theory of Metamaterials Design, in: dell'Isola, F., Steigmann, D. (Eds.), Discrete and Continuum Models for Complex (Meta) Materials, Cambridge University Press. pp. 141-196.

Dirrenberger, J., Forest, S., Jeulin, D., Colin, C., 2011. Homogenization of periodic auxetic materials. Procedia Engineering 10, $1847-1852$.

Drescher, A., de Josselin de Jong, G., 1972. Photoelastic verification of a mechanical model for the flow of a granular material. Journal of the Mechanics and Physics of Solids 20, 337-340.

Duan, S., Wen, W., Fang, D., 2018. A predictive micropolar continuum model for a novel three-dimensional chiral lattice with size effect and tension-twist coupling behavior. Journal of the Mechanics and Physics of Solids 121, 23-46.

Frenzel, T., Kadic, M., Wegener, M., 2017. Three-dimensional mechanical metamaterials with a twist. Science 358, $1072-1074$.

Geuzaine, C., Remacle, J.F., 2009. Gmsh: a three-dimensional finite element mesh generator with built-in pre- and post-processing facilities. International Journal for Numerical Methods in Engineering 79, 1309-1331.

Giorgio, I., dell'Isola, F., Misra, A., 2020. Chirality in 2D Cosserat media related to stretch-micro-rotation coupling with links to granular micromechanics. International Journal of Solids and Structures 202, 28-38.

Hall, S., Muir Wood, D., Ibraim, E., Viggiani, G., 2010. Localised deformation patterning in 2D granular materials revealed by digital image correlation. Granular Matter 12, 1-14.

Hansen, P., 2010. Discrete Inverse Problems: Insight and Algorithms. Fundamentals of algorithms series, Society for Industrial and Applied Mathematics (SIAM), Philadelphia, PA (USA). doi:10.1137/1.9780898718836.

Harrington, M., Lin, M., Nordstrom, K.N., Losert, W., 2014. Experimental measurements of orientation and rotation of dense 3D packings of spheres. Granular Matter 16, 185-191.

Hild, F., Misra, A., dell'Isola, F., 2021. Multiscale DIC applied to Pantographic Structures. Experimental Mechanics 61, $431-443$.

Hild, F., Roux, S., 2012. Digital image correlation, in: Rastogi, P., Hack, E. (Eds.), Optical Methods for Solid Mechanics. A Full-Field Approach, Wiley-VCH, Weinheim (Germany). pp. 183-228.

Holtzman, R., Silin, D., Patzek, T.W., 2010. Frictional granular mechanics: A variational approach. International Journal for Numerical Methods in Engineering 81, 1259-1280.

iDICs, Jones, E., Iadicola, M. (Eds.), 2018. A Good Practices Guide for Digital Image Correlation. International Digital Image Correlation Society (iDICs), DOI: 10.32720/idics/gpg.ed1.

Jean, M., Moreau, J.J., 1987. Dynamics in the presence of unilateral contacts and dry friction: A numerical approach, in: Del Piero, G., Maceri, F. (Eds.), Unilateral Problems in Structural Analysis — 2, Springer Vienna, Vienna. pp. 151-196.

Kuhn, M., Bagi, K., 2004. Contact rolling and deformation in granular media. International Journal of Solids and Structures 41, 5793-5820.

Larsson, S., Rodríguez Prieto, J., Gustafsson, G., Häggblad, H., Jonsén, P., 2020. The particle finite element method for transient granular material 


\section{Multiscalar DIC Analyses Reveal Non-standard Deformation Mechanisms}

flow: modelling and validation. Computational Particle Mechanics

Leclerc, H., Neggers, J., Mathieu, F., Hild, F., Roux, S., 2015. Correli 3.0. IDDN.FR.001.520008.000.S.P.2015.000.31500. Agence pour la Protection des Programmes, Paris (France).

Liu, X., Hu, G., Sun, C., Huang, G., 2011. Wave propagation characterization and design of two-dimensional elastic chiral metacomposite. Journal of Sound and Vibration 330, 2536-2553.

Liu, X., Huang, G., Hu, G., 2012. Chiral effect in plane isotropic micropolar elasticity and its application to chiral lattices. Journal of the Mechanics and Physics of Solids 60, 1907-1921.

Misra, A., Jiang, H., 1997. Measured kinematic fields in the biaxial shear of granular materials. Computers and Geotechnics 20, 267-285. Theoretical and Experimental Methods for Particulate Materials.

Misra, A., Lekszycki, T., Giorgio, I., Ganzosch, G., Müller, W., dell'Isola, F., 2018. Pantographic metamaterials show atypical poynting effect reversal. Mechanics Research Communications 89, 6-10.

Misra, A., Nejadsadeghi, N., De Angelo, M., Placidi, L., 2020. Chiral metamaterial predicted by granular micromechanics: verified with 1D example synthesized using additive manufacturing. Continuum Mechanics and Thermodynamics 32, $1497-1513$.

Nejadsadeghi, N., Misra, A., 2020. Extended granular micromechanics approach: a micromorphic theory of degree n. Mathematics and Mechanics of Solids 25, 407-429.

Poncelet, M., Somera, A., Morel, C., Jailin, C., Auffray, N., 2018. An experimental evidence of the failure of Cauchy elasticity for the overall modeling of a non-centro-symmetric lattice under static loading. International Journal of Solids and Structures 147, $223-237$.

Poorsolhjouy, P., Misra, A., 2019. Granular micromechanics based continuum model for grain rotations and grain rotation waves. Journal of the Mechanics and Physics of Solids 129, 244-260.

Reasa, D., Lakes, R., 2019. Ccosserat effects in achiral and chiral cubic lattices. Journal of Applied Mechanics 86, 111009.

Rechenmacher, A., 2006. Grain-scale processes governing shear band initiation and evolution in sands. Journal of the Mechanics and Physics of Solids $54,22-45$.

Réthoré, J., Roux, S., Hild, F., 2009. An extended and integrated digital image correlation technique applied to the analysis fractured samples. European Journal of Computational Mechanics 18, 285-306.

Richefeu, V., Combe, G., 2020. The particle image tracking technique: An accurate optical method for measuring individual kinematics of rigid particles. Strain 56, e12362.

Richefeu, V., Combe, G., Viggiani, G., 2012. An experimental assessment of displacement fluctuations in a $2 \mathrm{~d}$ granular material subjected to shear. Géotechnique Letters 2, 113-118.

Schneebeli, G., 1956. Une analogie mécanique pour les terres sans cohésion. Comptes Rendus de l’Academie des Sciences 243, $125-126$.

Spadoni, A., Ruzzene, M., Gonella, S., Scarpa, F., 2009. Phononic properties of hexagonal chiral lattices. Wave Motion 46, $435-450$.

Surjadi, J., Gao, L., Du, H., Li, X., Xiong, X., Fang, N., Lu, Y., 2019. Mechanical metamaterials and their engineering applications. Advanced Engineering Materials 21, 1800864.

Sutton, M., 2013. Computer vision-based, noncontacting deformation measurements in mechanics: A generational transformation. Applied Mechanics Reviews 65, 050802 .

Tikhonov, A., Arsenin, V., 1977. Solutions of ill-posed problems. J. Wiley, New York (USA).

Tomičević, Z., Hild, F., Roux, S., 2013. Mechanics-aided digital image correlation. Journal of Strain Analysis for Engineering Design 48, 330-343.

Turco, E., dell'Isola, F., Misra, A., 2019. A nonlinear Lagrangian particle model for grains assemblies including grain relative rotations. International Journal for Numerical and Analytical Methods in Geomechanics 43, 1051-1079. 
Vardoulakis, I., 2019. Cosserat Continuum Mechanics - With Applications to Granular Media. volume 87 of Lecture notes in applied and computational mechanics. Springer, Cham (Switzerland).

Wang, D., Nejadsadeghi, N., Li, Y., Shekhar, S., Misra, A., Dijksman, J., 2021. Rotational diffusion and rotational correlations in frictional amorphous disk packings under shear. Soft Matter 17, 7844-7852.

Wu, W., Hu, W., Qian, G., Liao, H., Xu, X., Berto, F., 2019. Mechanical design and multifunctional applications of chiral mechanical metamaterials: A review. Materials Design 180, 107950.

\section{Appendix B}

In the following, the results obtained for the extensional test are reported when the dotted surface was monitored (Figure 15).
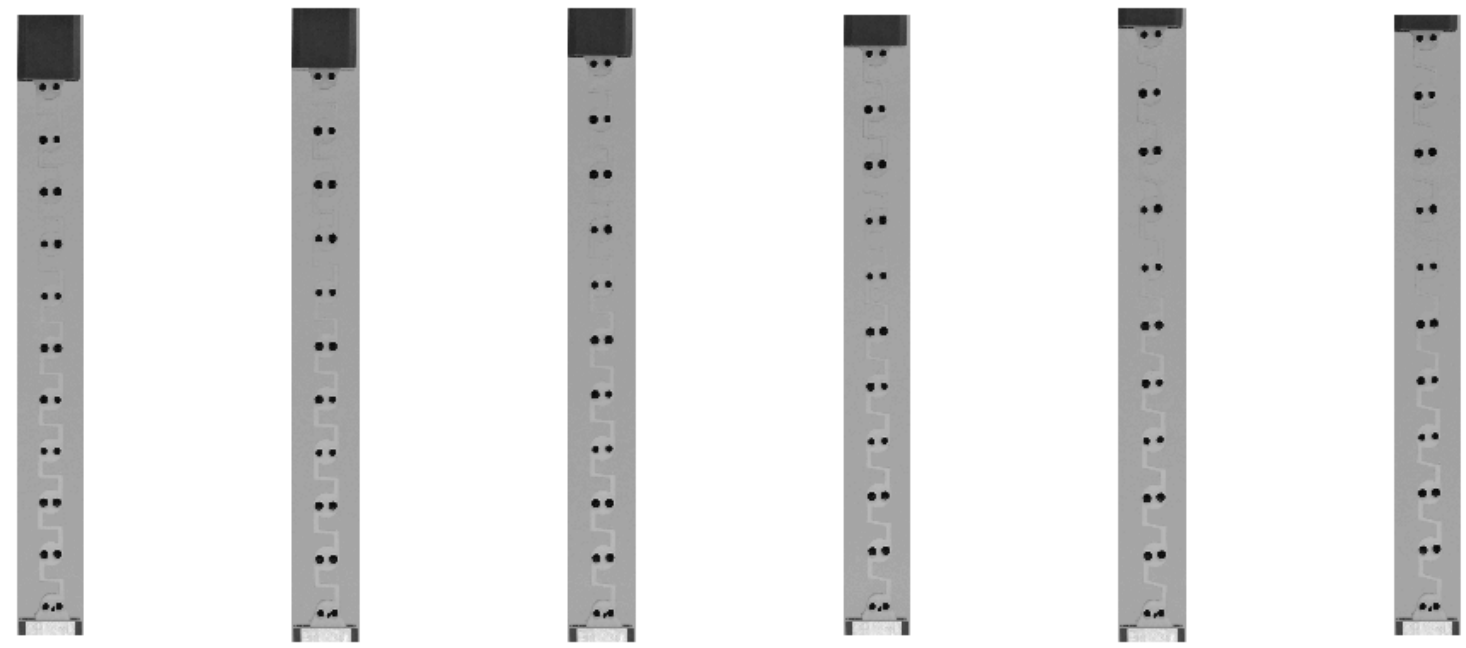

Figure 15: Cropped gray level images of the extensional test with dotted grains

\subsection{Multiscalar DIC Analyses}

Figure 16 show the three meshes for the convergence analysis at the macroscale. The element sizes were 91,37 , and 23 px. 

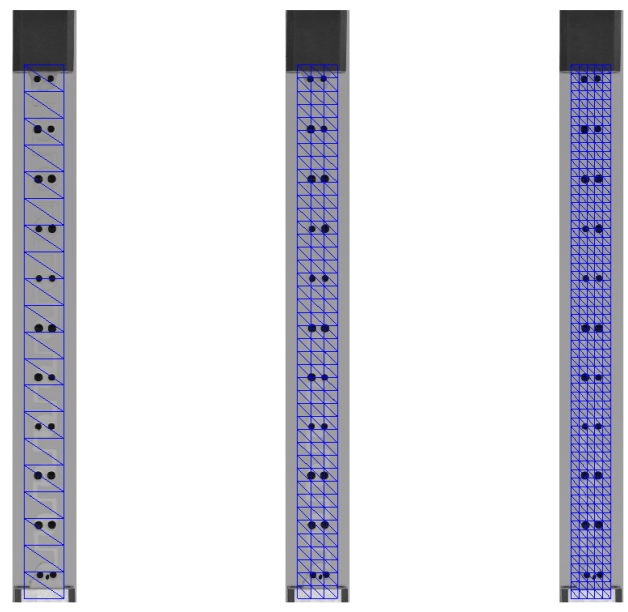

Figure 16: Three meshes used for the DIC analyses at the macroscale of the dotted (b) surface

Figure 17 shows that the RMS residuals are higher (i.e., between 1.5 and 5 times) than the level associated with acquisition noise (i.e., 1.2 gray level). They also grew as the applied displacement amplitude increased. The same remarks applied for the macroscale analyses of the speckled surface (Figure 6). This observation shows that, for both tests, the macroscopic kinematic bases were not able to properly capture the complexity of local displacement fields induced by the geometry of the metamaterial as the applied displacement increases.

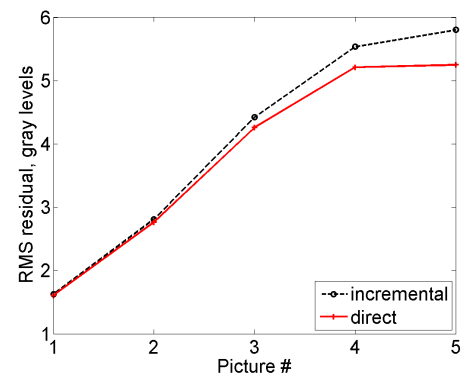

(a)

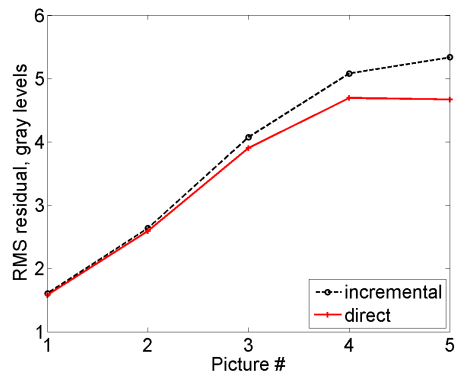

(b)

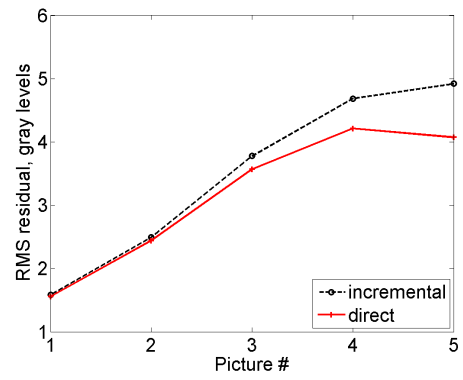

(c)

Figure 17: RMS gray level residuals for the three meshes of the dotted surface (Figure 16) and the two DIC routes 
When following the backtracking procedure for the dotted surface (Figure 18(leftmost)), the dots were also considered in the mask that was used for registration purposes (Figure 18(left)). The inclusion of dots within the mask is expected to provide more accurate registration. This could further improve the accuracy of the measured deformation fields especially in the lateral direction (transverse to the extension direction). It is noted that the accuracy of lateral deformations are of particular interest in this analysis (Misra et al., 2020). Figure 18(right)) shows the backtracked mesh. The same operation was carried out for the 4-element zones of discrete approaches following the motion of each dot (Figure 18(rightmost)).
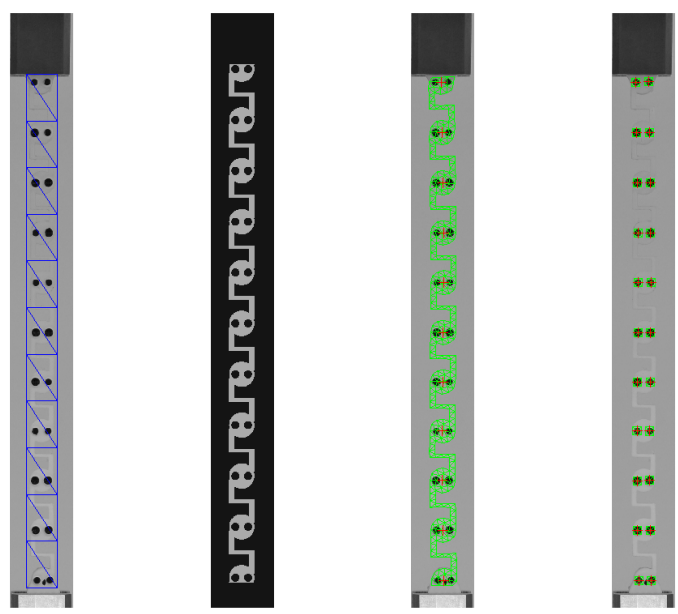

Figure 18: Backtracked meshes for microscale DIC of the dotted surface. The image of the reference configuration (leftmost) is registered with that of the nominal configuration (left) using the auxiliary mesh shown in blue. The backtracked mesh is laid over the reference image (right). The grain centers (red crosses) are also depicted. Similarly, the 4-element zones about each dot are also backtracked (rightmost)

The mean element size was equal to $16 \mathrm{px}$ for this last mesh. For the dotted surface, the RMS residuals were even lower (i.e., less than 2 times the level due to acquisition noise) than for the speckled surface (i.e., of the order of 2.3 the level due to acquisition noise). However, it should not be concluded that in that case the results are more trustworthy since the surface patterns were 
completely different. However, it can be concluded that in both cases, the kinematics is very well captured by such analyses. If deformation details were to be sought, the mesh should be further refined.

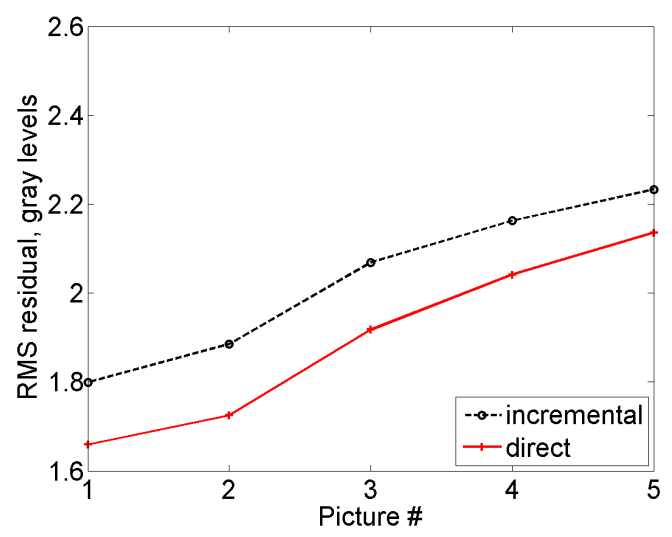

Figure 19: RMS gray level residuals for the DIC analyses at the microscale of the dotted surface

For the grain-scale analyses with 3 DOFs, a ratio 1.5 to less than 3 is observed for the RMS residuals when related to acquisition noise (Figure 20). These results prove that the selected kinematics is able to better capture the underlying motions of the grains. For the dotted surface, the 6 DOF analyses was not sought. Instead, each dot was individually tracked by considering 4-element zones arranged about each dot center. These analyses are far more delicate and sensitive when the element length is decreased because not much contrast is available apart from the dot edges. This remark explains why the incremental calculations were not able to converge to similar solutions as direct analyses. For the direct analyses, the RMS residuals did not vary much and were low enough (i.e., about 2.5 times the level associated with acquisition noise) to deem the measurements reliable. 


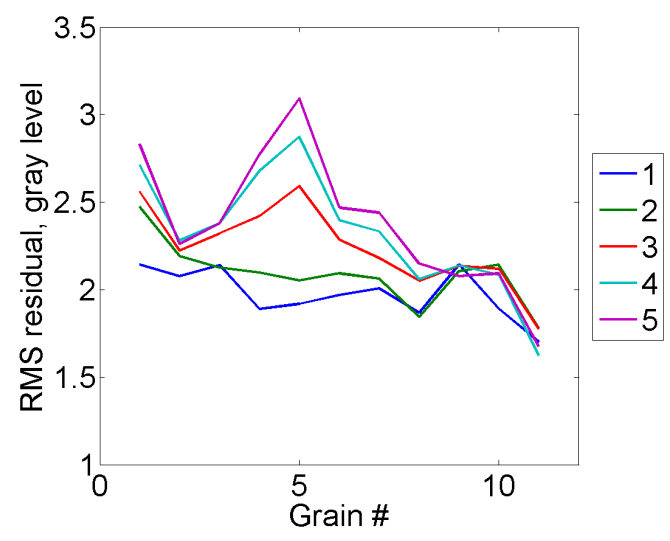

(a)

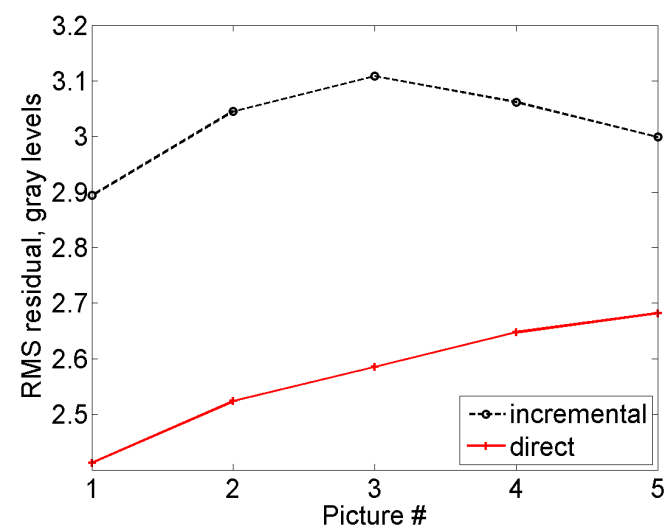

(b)

Figure 20: RMS gray level residuals for grain-scale (a) and local (b) DIC analyses of the dotted surface

\subsection{Results for the Dotted Surface}

The DIC results obtained with the different DIC approaches for the dotted surface are described. It is noted that these calculations are far more delicate as the surface contrast is no longer evenly distributed as in the previous case. However, the implemented DIC approach (using appropriate regularization) was able to extract many details of the deformation behavior as described in the following sections.

\subsubsection{Macroscale Analysis}

As mentioned above, the analyses at the macroscale were possible due to the regularization strategy used herein since most of the contrast is provided by the $2 \times 11$ black dots (Figure 21(leftmost)). Interestingly, even for the quality of the prepared dotted surface, the results are similar to those for the speckled surface. Consequently, the observation at the macroscale are the same as before. It is worth noting, though, that the contours of transverse displacements (Figure 21(left)) and longitudinal displacements once the mean strain contribution was taken out (Figure 21(right)) are inclined in the opposite direction. Similarly, the sign of the transverse displacements is also inverted. These two effects are due to the fact that the back face was observed instead of the front face. Clearly, the granular string is not invariant to mirror transformations about the vertical axis 

and this aspect is reflected in the sign of measured displacements.
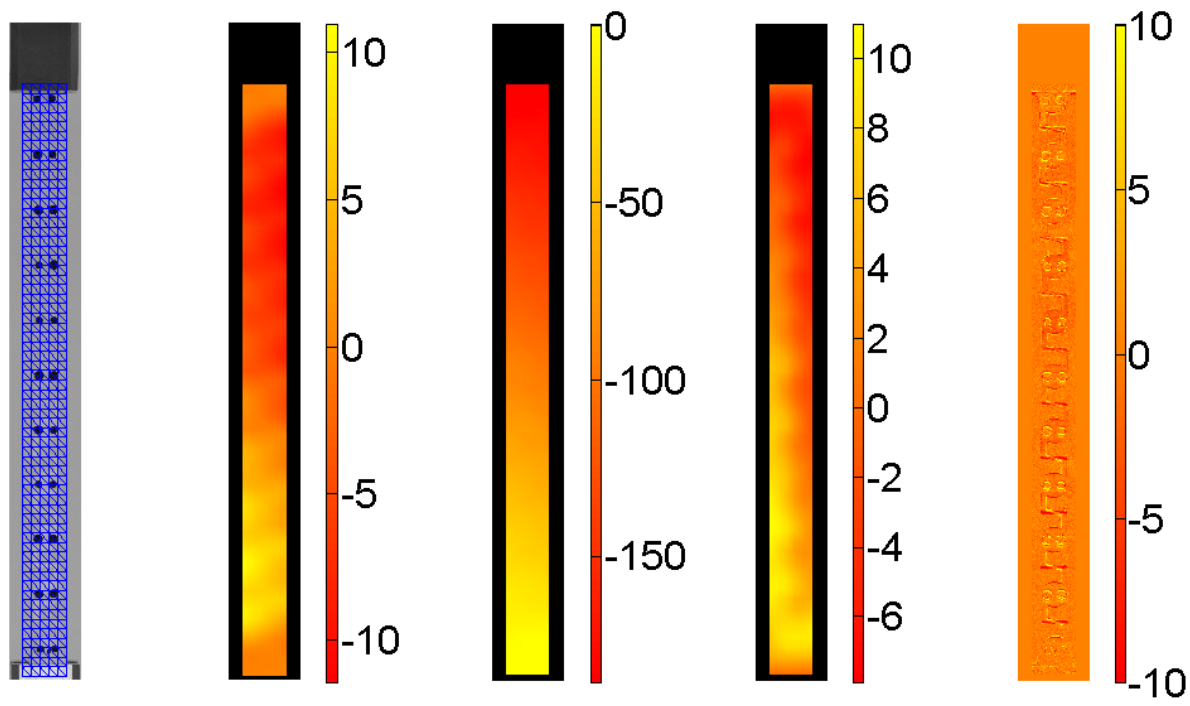

Figure 21: Mesh laid over the reference picture for the macroscale analysis of the dotted surface (leftmost). Transverse displacement (expressed in px) field for the last analyzed picture (left). Corresponding longitudinal displacement (expressed in px) field (middle) and displacement fluctuations once the mean strain contribution was taken out (right). Gray level residual field (rightmost)

\subsubsection{Microscale Analysis}

The general features observed for the transverse displacement field and from the longitudinal displacement fluctuations are confirmed by microscale analyses with 16 px elements (Figure 22). For the longitudinal displacement field, the same type of complexity is noted as for the speckle pattern. All these interpretations could be made since the gray level residuals remained very low even for such high displacement amplitudes. 

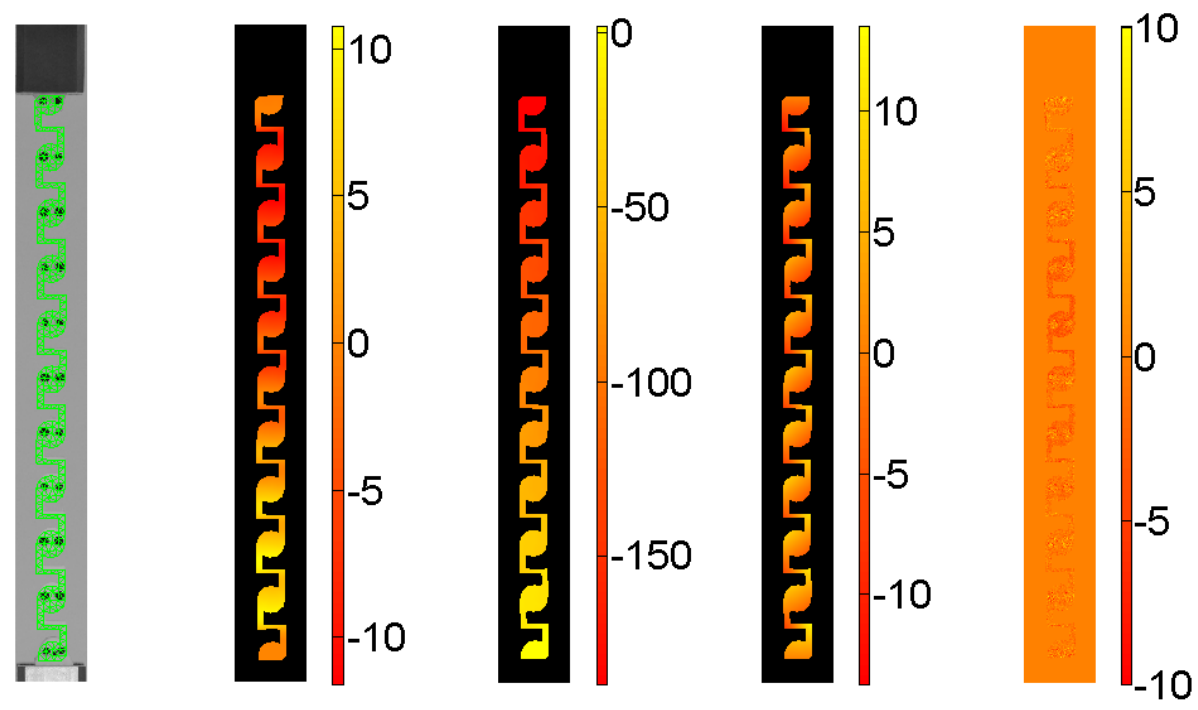

Figure 22: Mesh laid over the reference picture for the microscale analysis of the dotted surface (leftmost). Transverse displacement (expressed in px) field for the last analyzed picture (left). Corresponding longitudinal displacement (expressed in px) field (middle) and displacement fluctuations once the mean strain contribution was taken out (right). Gray level residual field (rightmost)

In addition, the strain fields shown in Figure 23 exhibit the same trends as observed for the speckle pattern (Figure 14) for a refined mesh with 3 px elements. In particular, identical deformation mechanisms and strain levels occurred in this new analysis. 

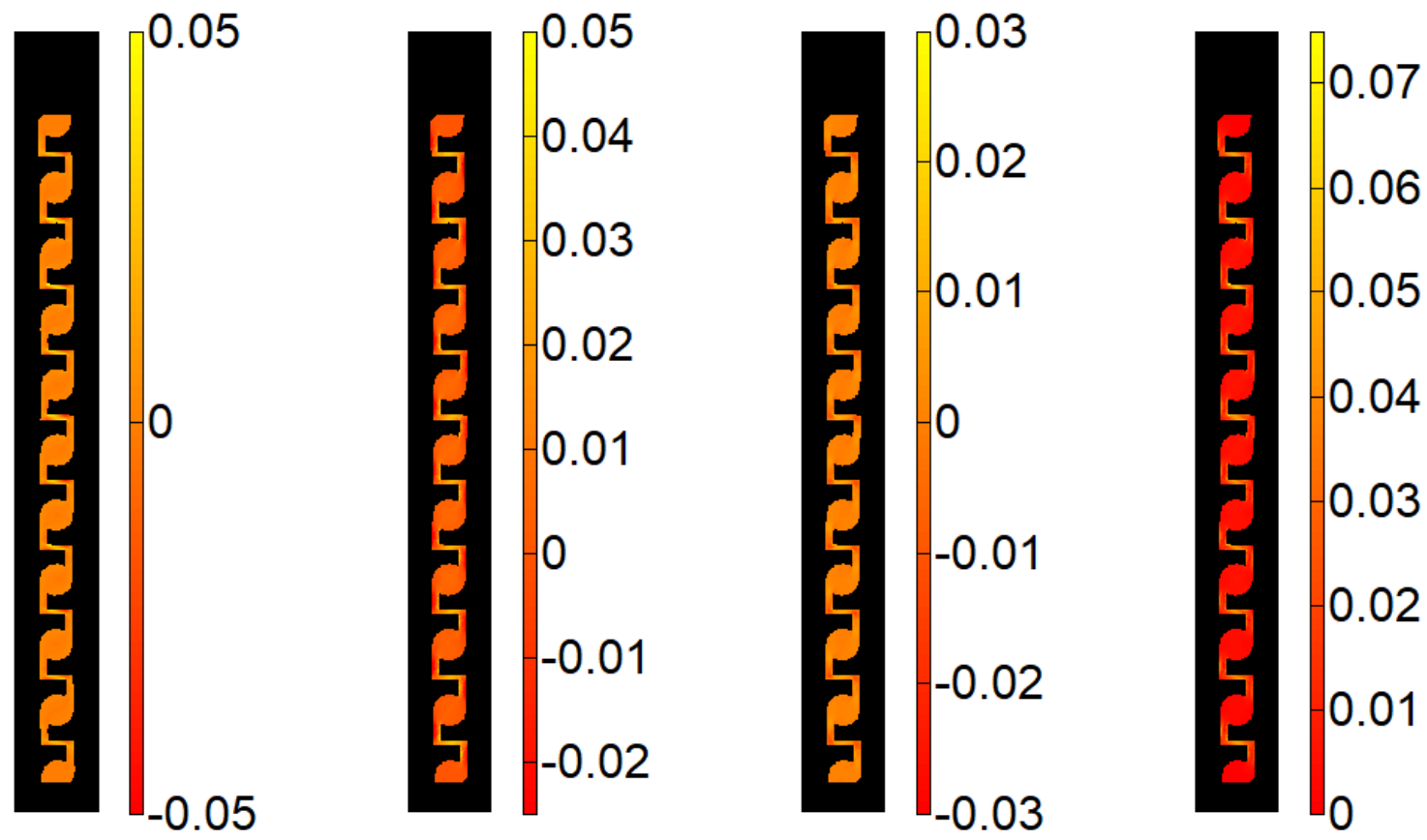

Figure 23: Transverse strain field for the last analyzed picture of the dotted surface (leftmost). Corresponding longitudinal (left), shear (right), and equivalent elastic strain field (rightmost)

\subsubsection{Mesoscale Analysis}

In Figure 24, the changes of the 3 DOFs per grain and for the 5 images are reported. It is worth noting that the maximum amplitude was as high as in the previous test for which 7 pictures were available. The present case is therefore even more challenging. However, thanks to the initialization strategy, convergence was fast and the residuals were sufficiently low to deem these results trustworthy. The transverse displacements are anti-symmetric with respect to the middle grain, which was not the case for the speckled surface (Figure 10). Similarly, the longitudinal displacement fluctuations are anti-symmetric as well in contrast to what was observed previously. Such differences can be explained by the fact that the dotted pattern corresponds to the first cycle, and the speckle pattern to the second cycle (opposite sides were monitored with a single camera). Some minor plasticity and damage may have occurred during these two cycles. The sample was unmounted between the two cycles (one week apart) so the application of boundary conditions may have changed a bit. Last, the misalignment of the camera in terms of verticality and the differences 

in the choice of coordinate system for the two DIC analyses may also have a small influence.

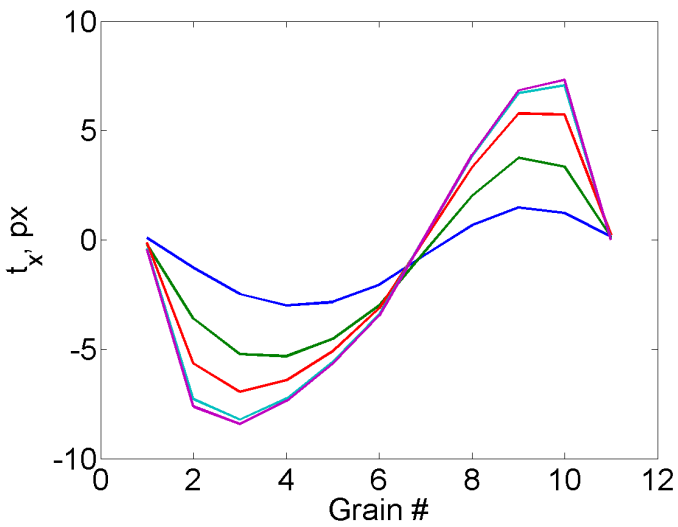

(a)

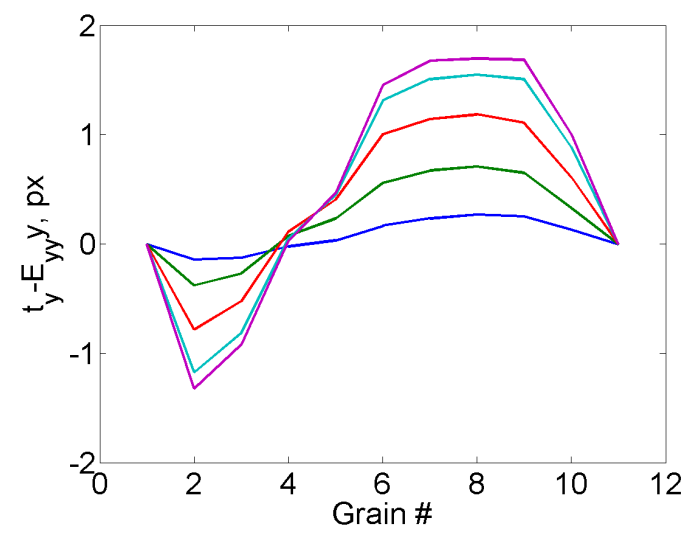

(c)

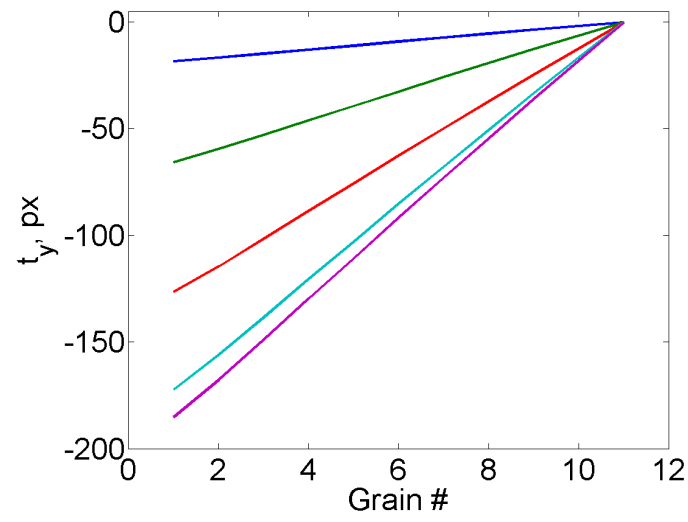

(b)

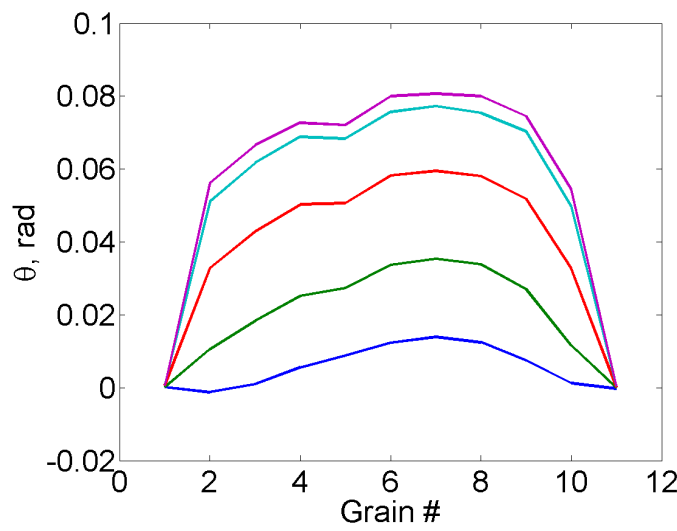

(d)

Figure 24: Change of the transverse translation (a), longitudinal translation (b), fluctuations of longitudinal translation, and rotation (d) of the eleven grains for all analyzed images of the dotted surface (for line color, refer to Figure 20(a))

The analysis at the grain scale could also be performed by only following the displacement of each dot center thanks to 4-element zones (Figure 25). The gray level residuals are very low, which again validates the reported measurements. All the trends observed at the microscale are again present in these last results. 


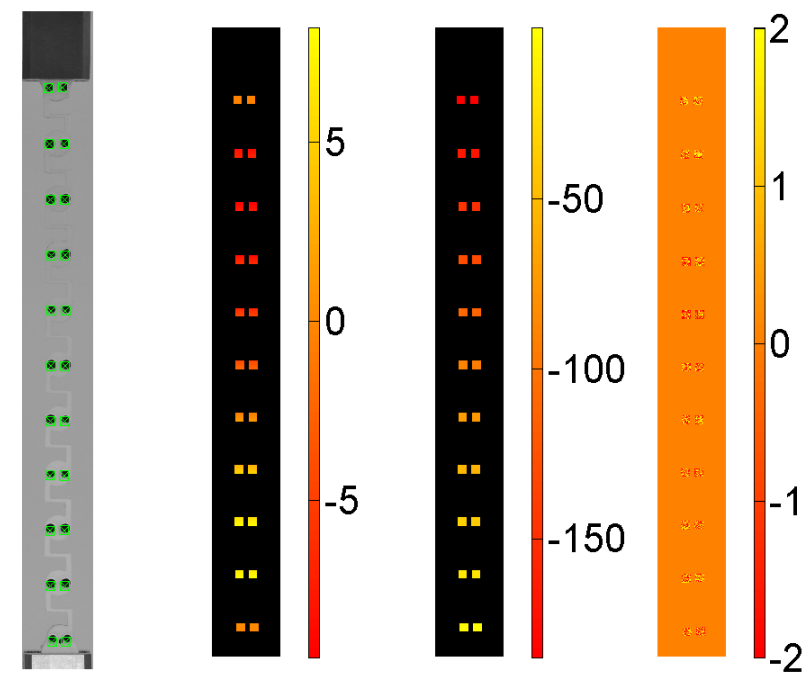

Figure 25: Mesh laid over the reference picture for the local analyses of the dotted surface (leftmost). Transverse displacement (expressed in px) field for the last analyzed picture (left). Corresponding longitudinal displacement (expressed in px) field (right) and gray level residual field (rightmost)

\section{Appendix B: DIC Framework}

Different parameterizations of the sought displacement field were considered (see Section 3.1) and are stated in a general form as

$$
\boldsymbol{u}(\boldsymbol{x},\{\boldsymbol{v}\})=\sum_{i} \Phi_{i}\left(\boldsymbol{x}, v_{i}\right)
$$

where $v_{i}$ is the degree of freedom (DOF) associated with the $i$-th trial displacement field $\boldsymbol{\Phi}_{i}\left(\boldsymbol{x}, v_{i}\right)$, and all DOFs are gathered in the column vector $\{\boldsymbol{v}\}$. In many cases, a linear dependence of the trial field with respect to the sought DOF may be assumed

$$
\boldsymbol{\Phi}_{i}\left(\boldsymbol{x}, v_{i}\right)=v_{i} \boldsymbol{\varphi}_{i}(\boldsymbol{x})
$$

with no summation over index $i$. This is for instance the case when seeking rigid body translations or using finite element discretizations of the displacement field. On the other hand, when large rigid body rotations are expected, then the corresponding displacement field $\Phi_{R B R}$ is given as

$$
\boldsymbol{\Phi}_{R B R}(\boldsymbol{x}, \theta)=[\boldsymbol{R}(\theta)-\boldsymbol{I}] \boldsymbol{x}
$$


where $[\boldsymbol{R}(\theta)]$ is the rotation matrix, which non-linearly depends upon the angle $\theta$, and $[\boldsymbol{I}]$ the identity matrix.

Let us introduce the kinematic sensitivities, namely, the partial derivatives of the trial displacement field $\boldsymbol{\Phi}_{i}$ with respect to the corresponding DOF $v_{i}$

$$
\boldsymbol{s}_{i}\left(\boldsymbol{x}, v_{i}\right)=\frac{\partial \boldsymbol{\Phi}_{i}}{\partial v_{i}}\left(\boldsymbol{x}, v_{i}\right)
$$

If the trial displacement field $\Phi_{i}$ is linearly dependent on its DOF $v_{i}$, then $\boldsymbol{s}_{i} \equiv \boldsymbol{\varphi}_{i}$, otherwise $\boldsymbol{s}_{R B R}(\boldsymbol{x}, \theta)=\partial[\boldsymbol{R}(\theta)] / \partial \theta \boldsymbol{x}$.

In the DIC analyses, the DOFs $\{\boldsymbol{v}\}$ are the unknowns to be determined. To this end, the sum of squared gray level residuals over the considered region of interest (ROI)

$$
\rho_{c}^{2}(\{\boldsymbol{v}\})=\sum_{\mathrm{ROI}} \rho^{2}(\boldsymbol{x},\{\boldsymbol{v}\})
$$

is minimized with respect to $\{\boldsymbol{v}\}$ (Hild and Roux, 2012). The pixel-wise gray level residual is given as

$$
\rho(\boldsymbol{x},\{\boldsymbol{v}\})=f(\boldsymbol{x})-g(\boldsymbol{x}+\boldsymbol{u}(\boldsymbol{x},\{\boldsymbol{v}\}))
$$

where $f$ represents the image in the reference configuration, and $g$ the image in the deformed configuration. The root mean square (RMS) residual computed over the ROI, $\varrho_{c}^{2}$, defines the overall registration quality associated with the selected kinematic basis (i.e., a measure of how well it reproduces the deformed configuration with respect to the reference).

The minimization of $\varrho_{c}^{2}$ is performed via Gauss-Newton iterations stated as follows

$$
\left[\boldsymbol{H}_{\boldsymbol{c}}\right]\{\partial \boldsymbol{v}\}=\left\{\boldsymbol{h}_{\boldsymbol{c}}\right\}
$$

where $\left[\boldsymbol{H}_{\boldsymbol{c}}\right]$ denotes the DIC Hessian

$$
\left(H_{c}\right)_{j k}=\sum_{\mathrm{ROI}}\left(\nabla \widetilde{g}(\boldsymbol{x}) \cdot \boldsymbol{s}_{j}(\boldsymbol{x})\right)\left(\nabla \widetilde{g}(\boldsymbol{x}) \cdot \boldsymbol{s}_{k}(\boldsymbol{x})\right)
$$

$\left\{\boldsymbol{h}_{\boldsymbol{c}}\right\}$ the DIC residual vector

$$
\left(h_{c}\right)_{j}=\sum_{\mathrm{ROI}}(f(\boldsymbol{x})-\tilde{g}(\boldsymbol{x}))\left(\boldsymbol{\nabla} \widetilde{g}(\boldsymbol{x}) \cdot \boldsymbol{s}_{j}(\boldsymbol{x})\right)
$$




$$
\varrho_{m}^{2}(\{\boldsymbol{v}\})=\{\delta \boldsymbol{v}\}^{\top}[\boldsymbol{K}]^{\top}[\boldsymbol{K}]\{\delta \boldsymbol{v}\}
$$

The minimization of augmented residual square $\rho_{t}^{2}=\varrho_{c}^{2}+w_{m} \varrho_{m}^{2}$ is performed via Gauss-Newton iterations as follows

$$
\left[\boldsymbol{H}_{\boldsymbol{c m}}\right]\{\partial \boldsymbol{v}\}=\left\{\boldsymbol{h}_{\boldsymbol{c}}\right\}-w_{m}[\boldsymbol{K}]^{\top}[\boldsymbol{K}]\{\delta \tilde{\boldsymbol{v}}\}
$$

with

$$
\left[\boldsymbol{H}_{\boldsymbol{c m}}\right]=\left[\boldsymbol{H}_{\boldsymbol{c}}\right]+w_{m}[\boldsymbol{K}]^{\top}[\boldsymbol{K}]
$$

where $\{\delta \widetilde{\boldsymbol{v}}\}$ denotes the current estimate of the incremental DOFs, and $[\boldsymbol{K}]$ the stiffness matrix associated with the chosen discretization. The weight $w_{m}$ multiplying the second cost function is proportional to a (regularization) length $\ell_{\text {reg }}$ raised to the power 4 (Réthoré et al., 2009; Tomičević et al., 2013). This length defines the physical size of the zone over which Hencky elasticity is 
assumed to apply. In the present case, this length should be compatible with the coarseness of the speckle pattern (Figure 15) and with the fact that the underlying hypothesis of Hencky elasticity was (at least partially) satisfied. Consequently, rather large regularization lengths were selected in the present cases.

With the selected regularization strategy (Tomičević et al., 2013), the weight $w_{m}$ is independent of the Young's modulus (provided it is positive). There is therefore no need for specifying it. Conversely, the stiffness matrix $[\boldsymbol{K}]$ is nonlinearly related to the Poisson's ratio of the medium. A value of $v=0.35$ was selected, which is that of the durable resin utilized herein. 